\title{
A Complete Survey on Automatically Diagnosing COVID-19 In the field of Computer Vision and A Collection of Medical Images
}

\author{
Aishwarza Panday \\ Stamford University Bangladesh \\ Dhaka, Bangladesh \\ aishipanday@gmail.com
}

\begin{abstract}
As COVID-19 is the source of millions of deaths throughout the world, it turned obligatory to fight against the COVID-19 pandemic. Due to the need for expensive equipment, experienced radiologists, and the time-consuming in Reverse Transcription Polymerase Chain Reaction (RT-PCR) test, researchers find out the necessity to embrace X-ray images and Computed Tomography (CT) images based diagnosing. Wreak havoc of COVID-19 instigated me to review current emerging Artificial Intelligence(Al) based automatic diagnosing models through the statistical survey that will pave out the way of research. In this paper, I study different available research resources at the time span from April 2020 to July 2020. In order to help researchers in further research, I presented a statistical survey so that researchers can pick a preeminent diagnosing model. I took a look at 74 papers from April to July and specified preprocessing techniques, feature extraction, classification method, interpretability method, and experimental result. Moreover, I analyze training,testing and validation split ratio, as well as look into the dataset's availability publicly. Some researchers are able to gain noticeable performance by adopting their own local model. On the contrary, some researchers adopt an existing pre-trained model and achieve the utmost result. Some models need to feed huge data and some models outperform despite having small data. In the following sections, all of the criteria will be illustrated briefly.
\end{abstract}

\section{Keywords: COVID-19 . Dataset Source . CT Images . X-ray Images . Feature Extraction}

\section{Introduction}

COVID-19 is the disease which is caused by severe acute respiratory syndrome coronavirus 2(SARS-CoV-2) that creates a worldwide pandemic of respiratory illness that emerged in Wuhan City, China in December 2019. The first recorded case of COVID-19 outside of China was on 13 January 2020. Within 17 days on 30 January, the World Health Organization(WHO) reported 7818 total confirmed cases worldwide among them China was in majority.WHO made the assessment that COVID-19 is a worldwide pandemic on 11 March 2020. From now on the number of affected persons and death is increasing day by day as it spreads from person to person.

Till now COVID-19 affected 213 countries $^{1}$ and the number of affected people reached $21,511,064$ the number of deaths is 766,394 up to 15 August 2020 globally. The globally recovered people's rate is $14,254,024$. Currently, Infected Patients are $6,490,646$ among them $99 \%$ is in mild condition and $1 \%$ is in critical condition.

In these circumstances diagnosing COVID-19 is inevitable otherwise the death toll will go on.RT-PCR is accepted as a standard diagnosing method widely but it only focuses on virus detection that can mislead to detect the people who have recovered from the virus. Moreover, it requires adequate expertise to collect viral RNA which is typically extracted from the nasopharyngeal of patients additionally RT-PCR is also time-consuming. To reach better model researchers focus on diagnosing based on scanning images(X-ray, CT-Scan). Researchers are paying more attention to Artificial Intelligence(AI) based diagnosing models as it requires less time, effort as well they focus on accuracy. RT-PCR test requires expensive equipment whereas image scanning based diagnosing is less expensive and doesn't need the

\footnotetext{
${ }^{1}$ https://www.worldometers.info/coronavirus/
} 
expertise to test. To make more accurate and precise results many researchers developed Domain Extension Transfer Learning CNN, transfer learning, attention mechanism, and Gradient-weighted Class Activation Mapping (Grad-CAM).

In this survey, many research papers will be reviewed; most of them are in pre-print format because diagnosing COVID-19 is beyond description. The motive behind this survey is to disclose numerous approaches that have been proposed and developed by researchers to create new probability and add a new direction to the research in Al-based diagnosing systems to combat against COVID-19.

Contribution of the survey is furnished as the following :

I successively reviewed and analyzed 74 papers that pay attention to COVID-19 diagnosing models from the perspective of preprocessing, feature extraction, classification, and evaluation among them 50 was X-ray image based diagnosing and 26 was CT-Scan based diagnosing, and 2 papers used both. These models are proposed from 1 April to 29 July [2] 2020. Based on the discussion of the existing model's feature extraction, I pointed out that model performance as well as point their classification methods and interpretability methods.

\section{Dataset Description:}

To get precise and accurate results more data is mandatory to feed the model. Relevant, accurate, and adequate training data is considered as the backbone of any diagnosing model. The more we will train our model the more accurate result it will produce. Based on different available research resources two types of datasets are found: computed tomography (CT) images and chest X-ray dataset.

Both of the chest X-ray and CT image datasets can be used to evaluate different feature extraction methods and their accuracy. In contrast to other types of pneumonia, it is asserted that both chest X-ray and CT image can help to point out the property of COVID-19, thus datasets can play a pivotal role in detecting COVID-19 and predicting survival rate from the disease. Due to the expense, shortage of training kits, inaccurate true positive rate, and time cost in RT-PCR test (X-ray image and CT image) based diagnosing is widely adopted to detect COVID-19 by the researchers that are based on the Artificial Intelligence(Al) system.

\subsection{CT images}

Computed tomography (CT) imaging is claimed as a substitute for the RT-PCR test. A precise image of the patient's chest is found from chest CT-Scan. An effective way to monitor the condition of the lung is Computed Tomography (CT) [8].COVID-19 CT segmentation dataset is evaluated in eight papers. Two papers evaluate their system using the COVID-CT dataset[29],[60]. Average $89 \%$ accuracy is gained by the researchers using this dataset. Particular details of the CT image data source are indicated in Table 1. Prediction comparison between COVID-19 and non-COVID is shown in Fig. 1 that demonstrates two chest CT images including COVID-19 affected and non-affected patients CT image. 


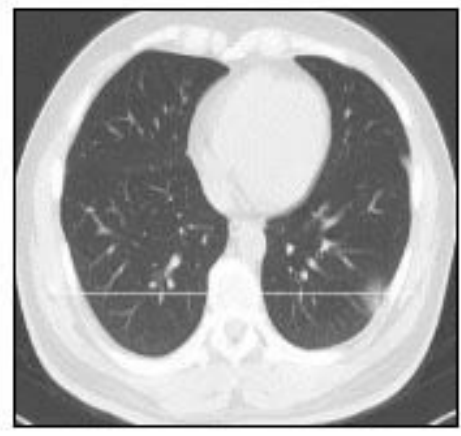

(a) COVID-19

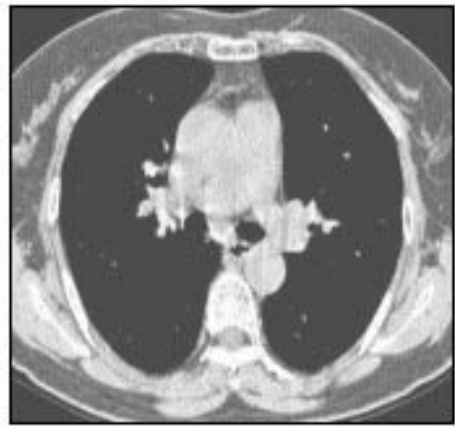

(b) Non Covid

Figure 1: Sample Chest CT Images ${ }^{2}$

Table 1 presents the available data sources that researchers used in their research to find out the promising model for detecting COVID-19 that requires less time as well as less effort. In addition Table 1 represents papers that make use of datasets along with a number of the papers.Besides, some papers utilized CT images datasource[4],[21],[42],[49],[58] that are not publicly available.Further in this section frequency of papers that used a specific dataset of CT images are illustrated in Fig. 2.

Table 1: Publicly available Dataset for CT Images

\begin{tabular}{|c|c|c|c|}
\hline Dataset Title & Available Source & Papers & $\begin{array}{c}\text { Number of } \\
\text { Papers }\end{array}$ \\
\hline $\begin{array}{l}\text { COVID-19 CT } \\
\text { segmentation } \\
\text { dataset }\end{array}$ & http://medicalsegmentation.com/covid19/ & $\begin{array}{c}{[2],[5],[8],[11],[50],[63],[65],} \\
{[39]}\end{array}$ & 8 \\
\hline SIRM & https://www.sirm.org/ & {$[46],[69]$} & 2 \\
\hline COVID-CT & https://github.com/UCSD-AI4H/COVID-CT & {$[60],[29]$} & 2 \\
\hline $\begin{array}{l}\text { University } \\
\text { Hospitals of } \\
\text { Geneva } \\
\text { (HUG) }\end{array}$ & $\begin{array}{c}\text { www.ChainZ.cn, El-Camino Hospital (CA), } \\
\text { Zhejiang Province, China University } \\
\text { Hospitals of Geneva (HUG) }\end{array}$ & [45] & 1 \\
\hline $\begin{array}{c}\text { Lung } \\
\text { Segmentation } \\
\text { and } \\
\text { Candidate } \\
\text { Points } \\
\text { Generation }\end{array}$ & $\begin{array}{c}\text { https://www.kaggle.com/arturscussel/lung- } \\
\text { segmentation-and-candidate-points-gener } \\
\text { ation }\end{array}$ & [49] & 1 \\
\hline LIDC & https://doi.org/10.1118/1.3528204 & [12] & 1 \\
\hline CC-19 & https://github.com/abdkhanstd/COVID-19 & [9] & 1 \\
\hline
\end{tabular}


Dataset

\section{Publicly available datasets used by the number of papers}

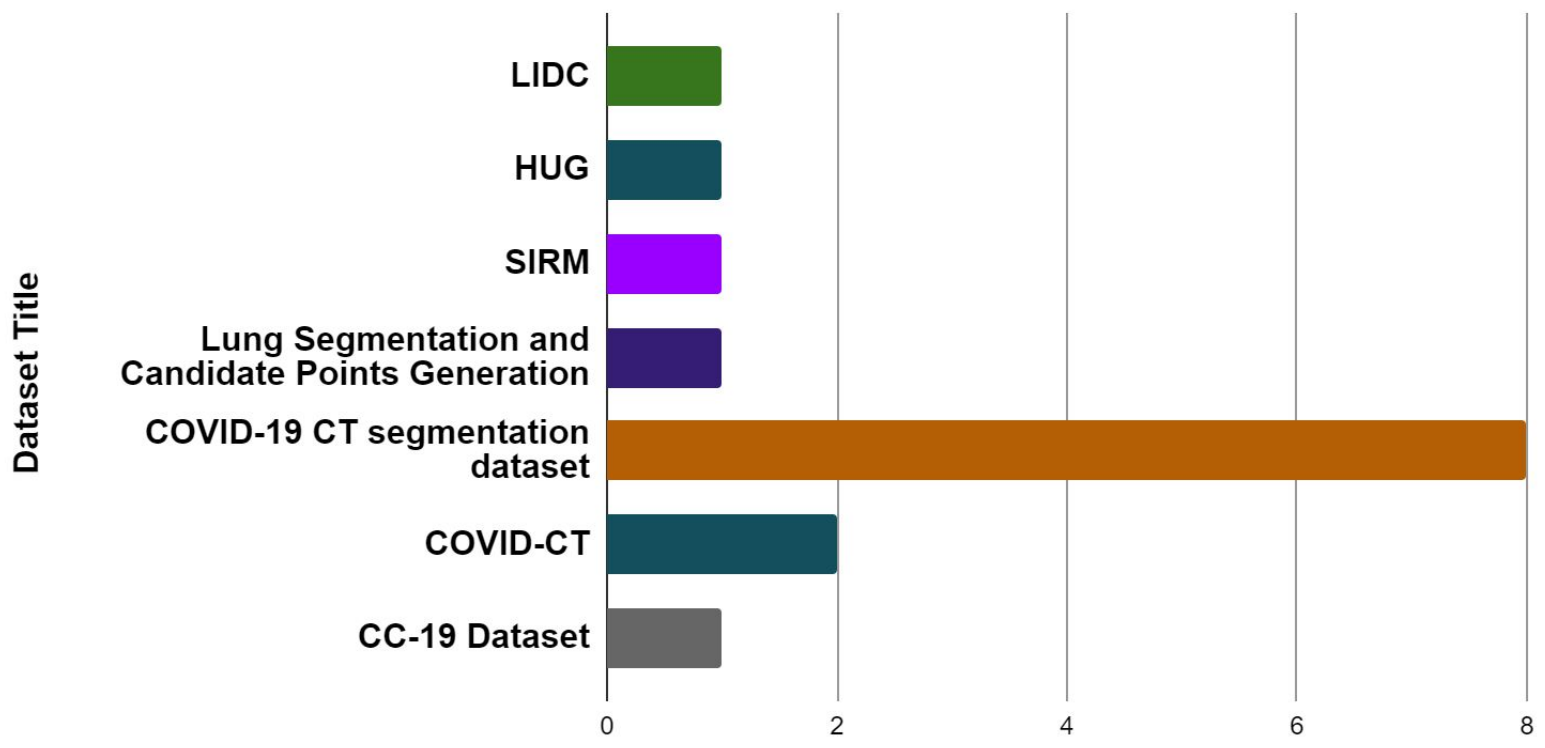

Number of papers

Figure 2: Frequency of papers that used a specific dataset of CT images

\subsection{X-ray images}

Due to the low sensitivity, a high false-negative rate of RT-PCR test, diagnosing using X-ray image gained high popularity because of its low expense, less time requirement, sufficient inexpensive equipment, high sensitivity. Twenty-five papers make use of COVID-Chest X-ray-dataset[3]. Chest X-ray Images (Pneumonia) dataset is evaluated by six papers. Evaluating COVID-19 Chest X-ray Dataset Initiative[36] in their system researcher gained accuracy 93\%. On the other hand, $97.3 \%$ accuracy is obtained using OCT and Chest X-ray images dataset in their model[10]. Precise details of available X-ray image data sources are presented in Table 2.Prediction comparison between COVID-19, normal, and viral pneumonia is shown in Fig. 3 that demonstrate three X-ray images. 


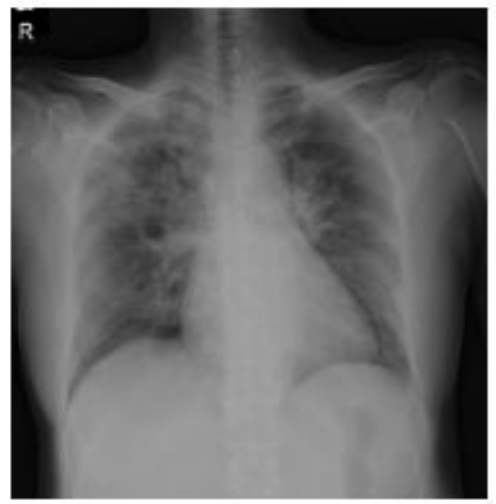

(a) COVID-19

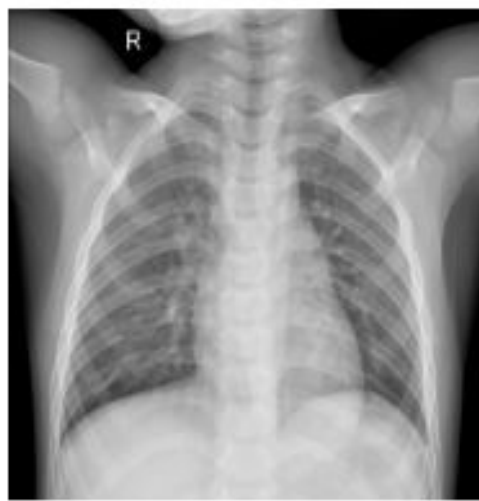

(b) Normal

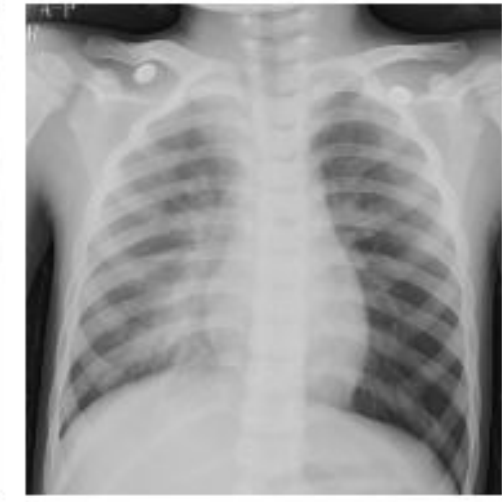

(c) Viral Pneumonia

Figure 3: Sample Chest X-ray Images ${ }^{3}$

Table 2 presents the available data sources that researchers used in their research to find out the promising model for detecting COVID-19 that requires less time as well as less effort. In addition Table 2 represents papers that make use of datasets along with a number of the papers.Besides, some papers utilized X-ray images datasource[41],[47],[49],[51],[52] that are not publicly available.Further in this section frequency of papers that used a specific dataset of X-ray images are illustrated in Fig. 4.

Table 2: Publicly available Dataset for X-ray Images

\begin{tabular}{|c|c|c|c|}
\hline Dataset Title & Available Source & Papers & $\begin{array}{c}\text { Number of } \\
\text { papers }\end{array}$ \\
\hline $\begin{array}{c}\text { Covid-chestxray-dat } \\
\text { aset }\end{array}$ & $\begin{array}{l}\text { https://github.com/iee } \\
\text { e8023/covid-chestxra } \\
\text { y-dataset }\end{array}$ & $\begin{array}{c}{[1],[3],[7],[10],[15],[17],[18],[20],[22],[23],[} \\
25],[31],[32],[40],[48],[49],[51],[54],[61], \\
{[66],[67],[70],[71],[72],[73]}\end{array}$ & 25 \\
\hline $\begin{array}{l}\text { Chest X-ray Images } \\
\text { (Pneumonia) }\end{array}$ & $\begin{array}{l}\text { https://www.kaggle.co } \\
\text { m/paultimothymooney } \\
\text { /chest-xray-pneumoni } \\
\text { a }\end{array}$ & {$[7],[18],[37],[54],[67],[76]$,} & 6 \\
\hline Radiopedia & $\begin{array}{c}\text { https://radiopaedia.or } \\
\text { g/articles/normal-ches } \\
\text { t-imaging-examples? } \\
\text { ang=gb }\end{array}$ & {$[17],[30],[33][51],[70]$} & 5 \\
\hline $\begin{array}{l}\text { COVID-19 } \\
\text { Radiography } \\
\text { Database }\end{array}$ & $\begin{array}{l}\text { https://www.kaggle.co } \\
\text { m/tawsifurrahman/cov } \\
\text { id19-radiography-data } \\
\text { base }\end{array}$ & {$[6],[24],[25],[36]$} & 4 \\
\hline $\begin{array}{l}\text { COVID-19 Chest } \\
\text { X-ray Dataset } \\
\text { Initiative }\end{array}$ & $\begin{array}{c}\text { https://github.com/agc } \\
\text { hung/Figure1-COVID- } \\
\text { chestxray-dataset }\end{array}$ & [36] & 1 \\
\hline
\end{tabular}

\footnotetext{
${ }^{3}$ https://github.com/drkhan107/CoroNet/blob/master/dataset
} 


\begin{tabular}{|c|c|c|c|}
\hline $\begin{array}{l}\text { ActualMed } \\
\text { COVID-19 Chest } \\
\text { X-ray Dataset } \\
\text { Initiative }\end{array}$ & $\begin{array}{l}\text { https://github.com/agc } \\
\text { hung/Actualmed-COV } \\
\text { ID-chestxray-dataset }\end{array}$ & {$[36]$} & 1 \\
\hline $\begin{array}{l}\text { COVID-19 Image } \\
\text { Data Collection }\end{array}$ & $\begin{array}{c}\text { https://arxiv.org/abs/2 } \\
003.11597\end{array}$ & {$[36],[43]$} & 2 \\
\hline $\begin{array}{l}\text { COVID-19 } \\
\text { database }\end{array}$ & $\begin{array}{c}\text { https://www.sirm.org/c } \\
\text { ategory/senza-catego } \\
\text { ria/covid-19/ }\end{array}$ & {$[1],[[24], 43],[51],[70]$} & 7 \\
\hline $\begin{array}{l}\text { Labeled Optical } \\
\text { Coherence } \\
\text { Tomography (OCT) } \\
\text { and Chest X-ray } \\
\text { Images for } \\
\text { Classification }\end{array}$ & $\begin{array}{l}\text { https://data.mendeley. } \\
\text { com/datasets/rscbjbr9 } \\
\text { sj/2. }\end{array}$ & [10],[15], [23], [44] & 3 \\
\hline $\begin{array}{l}\text { COVID-19 X-ray } \\
\text { dataset } \\
\text { (COVID-CAPS) }\end{array}$ & $\begin{array}{c}\text { https://github.com/Sh } \\
\text { ahinSHH/COVID-CAP } \\
\text { S }\end{array}$ & {$[57]$} & 1 \\
\hline $\begin{array}{c}\text { Kaggle RSNA } \\
\text { Pneumonia } \\
\text { Detection Dataset }\end{array}$ & $\begin{array}{l}\text { https://www.kaggle.co } \\
\text { m/c/rsna-pneumonia- } \\
\text { detection-challenge }\end{array}$ & {$[17],[22],[36],[61],[66],[72],[73]$} & 7 \\
\hline $\begin{array}{c}\text { NIH Chest X-ray } \\
\text { Dataset }\end{array}$ & $\begin{array}{c}\text { https://www.kaggle.co } \\
\text { m/nih-chest-xrays/dat } \\
\text { a }\end{array}$ & {$[16],[70]$} & 2 \\
\hline $\begin{array}{l}\text { Pneumonia } \\
\text { Classification }\end{array}$ & $\begin{array}{c}\text { https://drive.google.co } \\
\text { m/open?id=1J9l-pPtP } \\
\text { fLRGHJ42or4pKO2Q } \\
\text { ASHzLkkj }\end{array}$ & [33] & 1 \\
\hline COVID-19 & $\begin{array}{c}\text { https://github.com/mu } \\
\text { hammedtalo/COVID-1 } \\
9\end{array}$ & {$[16]$} & 1 \\
\hline COVIDGR-1.0 & $\begin{array}{l}\text { https://github.com/ari- } \\
\text { dasci/covidgr }\end{array}$ & [13] & 1 \\
\hline $\begin{array}{l}\text { pulmonary-chest-x-r } \\
\text { ay }\end{array}$ & $\begin{array}{l}\text { https://www.kaggle.co } \\
\text { m/kmader/pulmonary- } \\
\text { chest-xray-abnormaliti } \\
\text { es?select=ChinaSet_- } \\
\text { AllFiles }\end{array}$ & {$[7]$} & 1 \\
\hline $\begin{array}{l}\text { OCT and Chest } \\
\text { X-ray images }\end{array}$ & $\begin{array}{l}\text { https://data.mendeley. } \\
\text { com/datasets/rscbjbr9 } \\
\text { sj/2. }\end{array}$ & [10] & 1 \\
\hline
\end{tabular}




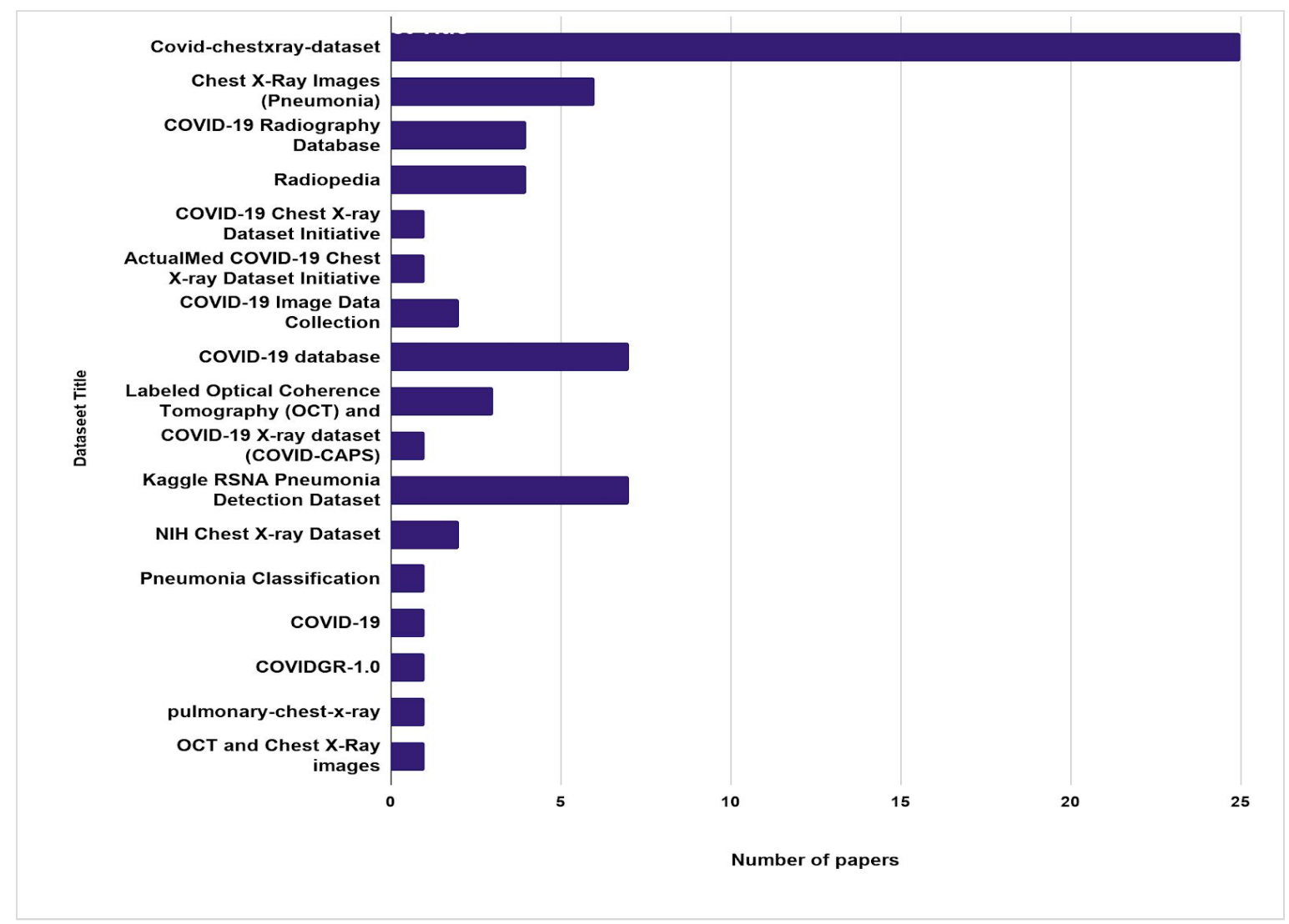

Figure 4: Frequency of papers that used a specific dataset of CT images

\section{Dataset Preprocessing :}

Data preprocessing regards as a strategy that converts raw data into prepared data. By data preprocessing methods researchers develop their data set as a clean and organized dataset. Thus, the performance of the diagnosing model also enhanced adopting various dataset preprocessing techniques. Data preprocessing is considered obligatory for diagnosing as it fills missing values, smooth noisy data indicates outliers, and sorts out inconsistencies. When researchers face difficulties because of the shortage of sample datasets, preprocessing can be a great help to the researchers by generating images. By dataset preprocessing before feature extraction diagnosing models can be generalized. In this section, adopted preprocessing methods are reviewed that is considered as the first step in diagnosing the model. Different types of preprocessing techniques like $224 \times 224$ resizing, brightness adjustment, Generative Adversarial Network(GAN), and Vertical flip are depicted in Fig.5, and from that figure clear visualization of various preprocessing techniques can be apprehended 

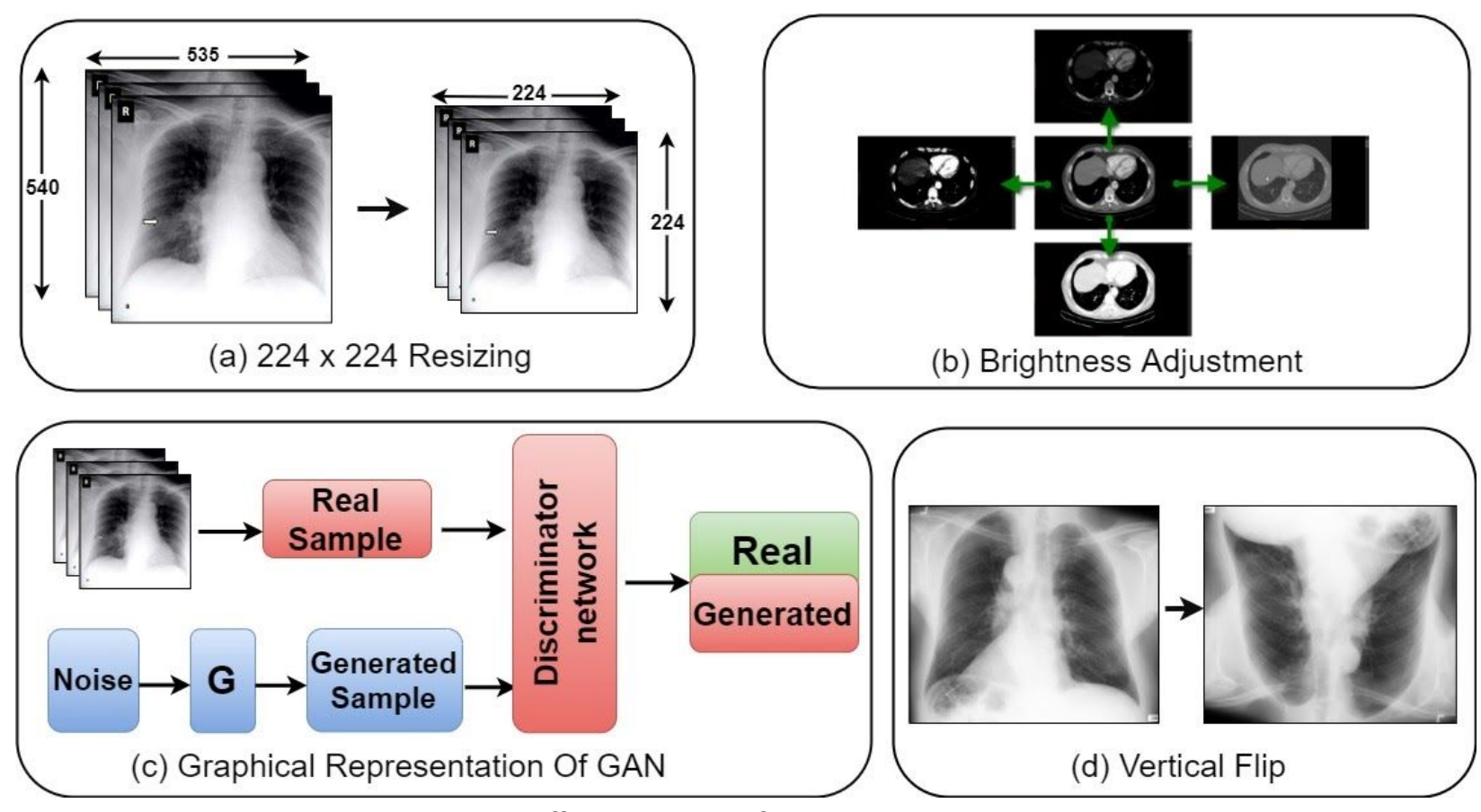

Figure 5: Different Types of Preprocessing Methods

Resizing is necessary to sustain images in the same estimate because the images are not always within the same size. All the samples in a dataset must be in the same size otherwise it postulates issues while diagnosing. Image resizing is done on different scales, sometimes at $352 \times 352$ [8] pixels and sometimes at Image size $237 \times 237$ pixels [10].

Flipping or Rotating performs image enhancement in datasets. Vertically or horizontally image pixels can be reversed. Additionally, images can be rotated at different angles for instance: 90, 180, or 270 degrees.

Cropping or Scaling is performed by resampling the image thus the size of the whole image changes. Scaling can enlarge or lessen an image in size. Cropping reduces redundancy and shrinks unwanted areas on the various scales. Researchers many times use random cropping in various scales [21],[55].

Brightness or Intensity adjusting increases or reduces the brightness of an image by substituting pixel values with a constant. Brightness can be increased or reduced by subtraction or addition.

Generative Adversarial Network(GAN): Due to the scarcity of Chest X-ray and CT image dataset GAN is widely adopted in several datasets to generate images at the same time it demises overfitting. Sometimes interference caused by image enhancement using GAN, to demise interference researchers performed histogram equalization [47] on the datasets. However, histogram equalization may impact on image details and show unexpected noise to resolve the problem Contrast Limited Adaptive Histogram Equalization (CLAHE) was proposed in paper [41].

In this section, I review preprocessing methods that are depicted in Table 3 used by researchers in their paper including resizing, flipping or rotating, scaling or cropping, contrast adjusting, brightness, or intensity adjusting, GAN, and so on. Resize a common feature of data preprocessing used in 31 papers. Next to it, Flipping is used in 29 papers. Along with that scaling or cropping, Contrast adjusting, Brightness or Intensity adjusting, and GAN is used in 20,14,7,4 papers respectively. The author used both resizing, flipping or rotating, and scaling or cropping in paper [2], and in paper [21] author used four preprocessing methods respectively Flipping or Rotating, Scaling or Cropping, Contrast adjusting, and Brightness or Intensity adjusting. Like this many authors used more than one preprocessing method in their research. Oppositely some authors used only one preprocessing method as an example in paper[9] author used scaling, in paper [11] the author used resizing $352 \times 352$, in paper [16] author used rotation, 
and so on. Moreover, some researchers also use an adaptive winner filter for noise reduction [59], Affine Transformation [31] in their research.

Table 3: Different types of preprocessing methods used by the papers

\begin{tabular}{|c|c|c|}
\hline $\begin{array}{l}\text { Preprocessing } \\
\text { Methods }\end{array}$ & Papers & $\begin{array}{l}\text { Number of } \\
\text { Papers }\end{array}$ \\
\hline Resize & $\begin{array}{l}{[1],[2],[5],[6],[8],[10][11],[16],[18],[19],[20],[22],[23][24],,[[26],[28],[} \\
30],[32],[37], 43],[45],[46],[48],[50],[53],[54],[56],[60],[61],[63],[67]\end{array}$ & 31 \\
\hline Flipping or Rotating & $\begin{array}{c}{[1],[2][15],[16],[21],[22],[24],[25],[27],[35],[36],[37],[38],[42],[43],[4} \\
6],[47],[48],[50],[51],[53],[55],[57],[61],[62],[65],[71],,[44]\end{array}$ & 29 \\
\hline Scaling or Cropping & $\begin{array}{c}{[2],[9],[18],[19],[21],[22],[25],[36],[46],[48],[50],[53],[55],[56],} \\
{[57],[60],[61],[65],[71],[74]}\end{array}$ & 20 \\
\hline Contrast adjusting & $\begin{array}{c}{[6],[21],[24],[28],[30],[35],[38],[41],[43],[48],[52],[53],[59],} \\
{[68]}\end{array}$ & 14 \\
\hline $\begin{array}{l}\text { Brightness or Intensity } \\
\text { adjusting }\end{array}$ & {$[21][36][46],[48],[52],[71],[74]$} & 7 \\
\hline GAN & [13] [39],[44], [60] & 4 \\
\hline
\end{tabular}

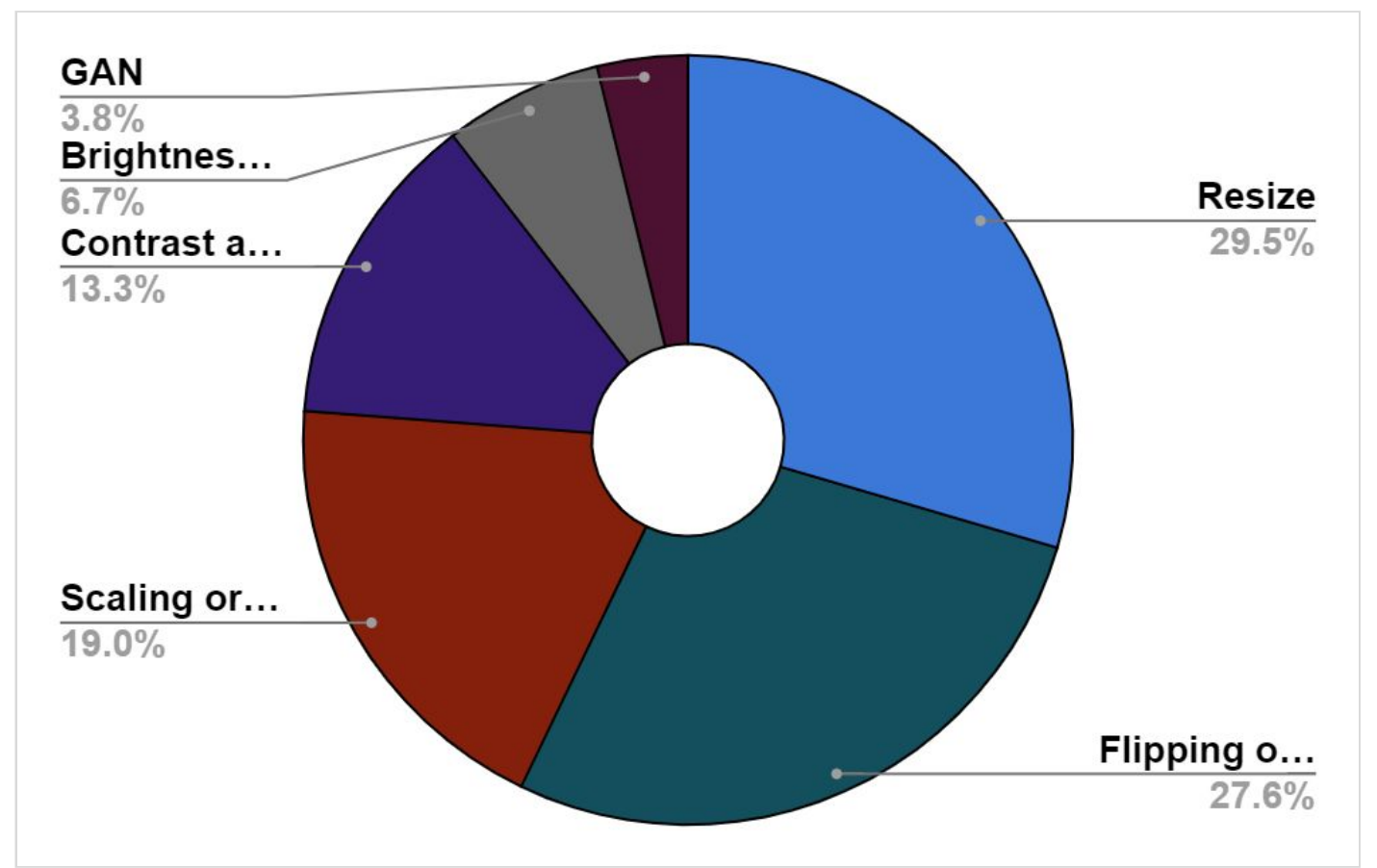

Figure 6:Ratio of Different preprocessing methods 


\section{Image Feature Extraction:}

Feature extraction is a procedure that classifies and recognizes features of images. Several images preprocessing techniques like resizing, normalization, scaling, etc are needed to apply on the sampled image before getting features using the extraction method. Feature extraction is beneficial for reducing the number of resources required for processing without dropping important or admissible information as well as Feature extraction can also lessen the amount of dispensable data. Convolutional Neural Network $(\mathrm{CNN})$ is used for both feature extraction and classification. Pertaining convolutional and pooling layer Feature extraction is carried out. A CNN architecture is instantiated in Fig. 7 has the following layers: Convolution, Pooling, Fully Connected layer. Except for CNN other pre-trained models as example ResNet, VGG, Inception and so on researchers also applied in their literature.

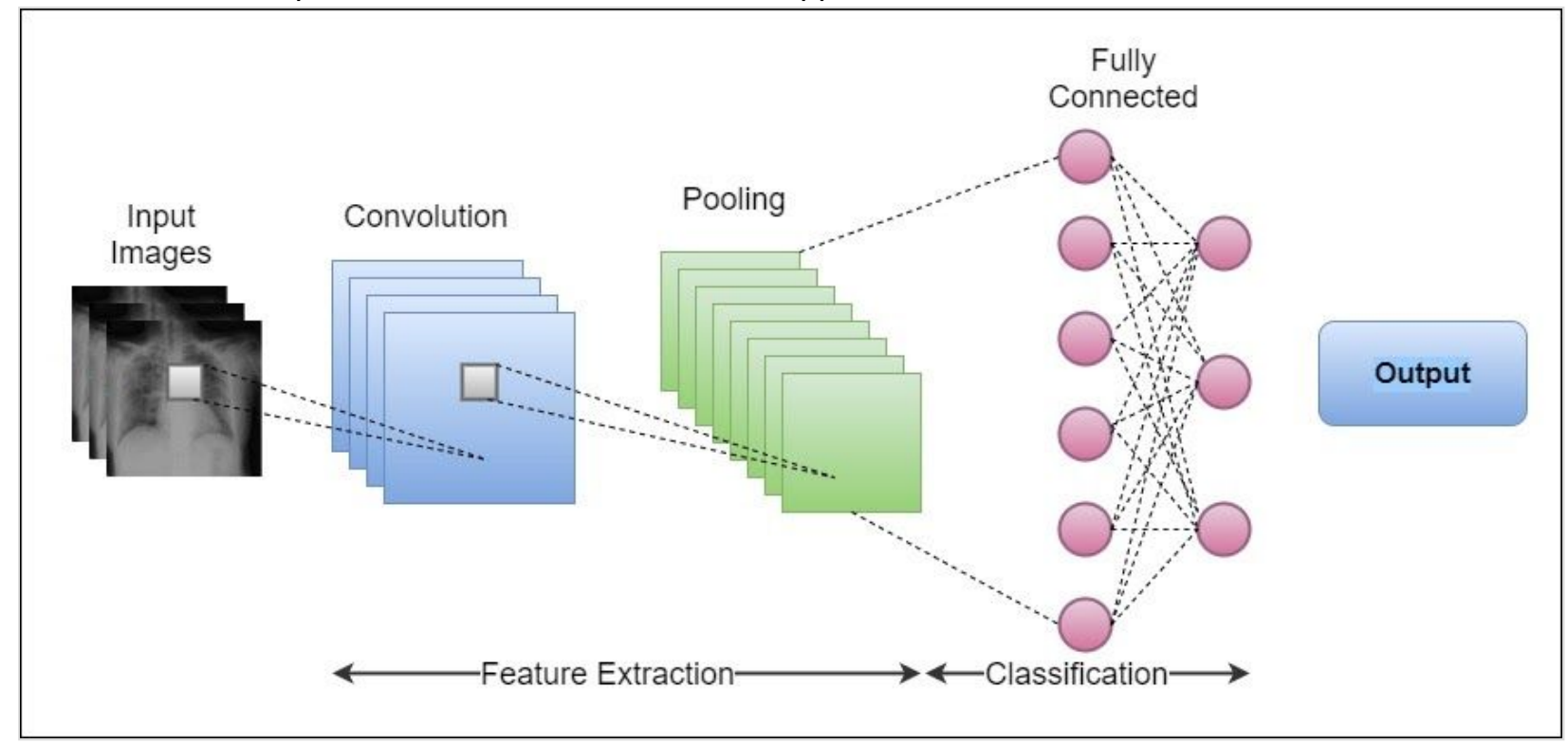

\subsection{Feature Extraction methods for CT}

Figure 7: Basic Pre-trained CNN architecture

To recognize patterns from the CT images between COVID-19 patients and nonaffected persons, various pertained models(ResNet, VGG, DenseNet, etc) availed the researchers nowadays. A few pre-trained models mostly used by researchers are delimited in Table 4, among all ResNet is used largely then VGG, DenseNET, and others.

Researchers use ResNet as a pre-trained model [42] for binary classification of CT scan images that is 71 layers deep and requires an input image size of $224 \times 224 \times 3$. As an extension of CNN researchers uses DenseNet [29] to boost computational efficiency reducing image dimension and obtain $90.61 \%$ accuracy.As a feature extraction method researchers employ a modified version of inception V3 (IV3*) then train the extracted features using layers of the capsule network [9] that achieved the highest sensitivity and lowest specificity compared to other pre-trained models. Researchers use eight different Deep Learning Models in the paper [4] and found NasNet and MobileNet performed better than the other six models.

Table 4: Feature Extraction methods for CT images used by the papers

\begin{tabular}{|c|c|c|}
\hline CNN & Paper & Number of Papers \\
\hline ResNet & {$[4],[9],[12],[21],[29][39],[42],[45],[46],[55],[58]$} & 11 \\
\hline VGG & {$[4],[8],[9],[11],[46],[55],[58]$} & 7 \\
\hline
\end{tabular}




\begin{tabular}{lll}
\hline DenseNet & {$[4],[9],[21],[29],[58]$} & 5 \\
\hline Mobilenet & {$[4],[9],[29]$} & 3 \\
\hline SqueezeNet & {$[42],[69]$} & 2 \\
\hline Inception & {$[29],[68]$} & 2 \\
\hline CrNet & {$[58]$} & 1 \\
\hline EfficientNet & {$[58]$} & 1 \\
\hline GoogLeNet & {$[46]$} & 1 \\
\hline InceptionResNet & {$[4]$} & 1 \\
\hline NasNetMobile & {$[4]$} & 1 \\
\hline FCN8 & {$[8]$} & 1 \\
\hline U-Net & {$[5]$} & 1 \\
\hline Alex-Net & {$[9]$} & 1 \\
\hline
\end{tabular}

Excluding those feature extraction methods researchers also use Conditional Generative Adversarial Network(cGAN)-based COVID-19 CT image synthesis method [2], Visualizes features via t-SNE, Least Absolute Shrinkage and Selection Algorithm (LASSO) [12] were used to find the most discriminative 12 features in distinguishing COVID-19 from other pneumonia (Three additional features were also extracted for the attentional regions, distance feature, 2D margin fractal dimension, and 3D grayscale mesh fractal dimension).In one more study researchers use Handcrafted features[34] Volume features, Infected lesion number, Histogram distribution, Radiomics features - Adaptive Feature: (Random forest + feature selection $)^{*} 2$ in their research. Additionally, I observe the use of Inception Recurrent Residual Neural Network(IRRCNN) [49] in another research, and attention mechanism-resdil block, and deep supervision [63]. Researchers trained their model with a linear activation for the output and a mean squared error for the loss function (loss1) and accuracy[64].

\subsection{Feature Extraction Methods for X-ray}

In this section several pertained models(ResNet,VGG,DenseNet etc) to detect COVID-19 from X-ray images are reviewed.A few pre-trained models mostly used by researchers are delimited in Table 5, among all ResNet is used largely then DenseNET, VGG, Inception, Xception, and others.

Researchers use 14 individual state-of-the-art pre-trained networks [7] in order to extract the deep features from the pre-trained networks and achieve the highest accuracy in ResNet as well as estimate time for feature extraction where ResNet requires less time for deep feature extraction among all 14 models. Convolution Neural Networks were trained using the supervised learning process for instance: Xception ResNet and VGG-16 [10] to minimize the loss function and obtain accuracy above $97 \%$ through VGG. Using 8 different Deep Learning Model [4] researchers found NasNet and MobileNet outperformed all the models and they used both CT image and X-ray images to evaluate their system.DenseNets concatenates output features rather than sum the output feature maps of the layer with the incoming feature maps.DenseNet outperformed than other models [18] having $85 \%$ sensitivity.In the following section, some other feature extraction methods adopted by the researchers are discussed elaborately. 
Table 5: Feature Extraction methods for X-ray images used by the papers

\begin{tabular}{|c|c|c|}
\hline CNN & Papers & $\begin{array}{l}\text { Number of } \\
\text { Papers }\end{array}$ \\
\hline ResNet & $\begin{array}{l}{[1],[4],[7],[10],[13],[14],[18],[20],[22],[27],[28],[31],[32],[38],[40],[41],[44],[} \\
47],[51],[61],[62],[66],[67],[71],[73],[74]\end{array}$ & 26 \\
\hline DenseNet & $\begin{array}{l}{[1],[4],[7],[14],[18][22],[23],[28],[30],[31],[41],[47],[48],[52],[56],[66],[67],[7} \\
\text { 2],[74] }\end{array}$ & 19 \\
\hline VGG & {$[1],[4],[7],[10],[16],[19],[27],[28],[30],[37],[40],[47],[51],[56],[66],[67],[71]$} & 17 \\
\hline Inception & {$[1],[14],[18],[24],[32],[41],[56],[66],[67]$} & 9 \\
\hline Xception & {$[7],[10],[15],[30],[54],[56],[61],[66],[67]$} & 9 \\
\hline InceptionResNet & {$[4],[7],[18],[22],[30],[56],[66],[67]$} & 8 \\
\hline NASnet & {$[4],[7],[22],[23],[30],[67]$} & 6 \\
\hline AlexNet & {$[7],[44],[66],[17],[27],[28]$} & 6 \\
\hline GoogLeNet & {$[7],[27],[40],[44],[66]$} & 5 \\
\hline SqueezeNet & {$[41],[44],[52],[62]$} & 4 \\
\hline ShuffleNet & {$[7],[52],[66]$} & 3 \\
\hline Mobilenet & {$[4],[7],[30]$} & 3 \\
\hline EfficientNet & [71] & 1 \\
\hline
\end{tabular}

Excluding those feature extraction methods researchers also use Parallel-Dilated COVIDNet (PDCOVIDNet) [1] to detect COVID-19 from chest X-ray images that can encapsulate and propagate important features in parallel over the network which boosts detection accuracy significantly. A multiple loss based deep neural network labeled as CovMUNET that is also proposed by researchers [3] to learn better feature maps and with the help of learning categorize the CXR images more precisely. In one more study, researchers use Fast Deep Learning Computer-Aided Diagnosis(CAD) System [25] against the Novel COVID-19 pandemic from Digital Chest X-ray Images that is based on YOLO Predictor. Additionally, I observe the use of Self Supervised Super Sample Decomposition for Transfer learning (4S-DT) [27], Gravitational Search Algorithm (GSA) based model [48], Inception Recurrent Residual Neural Network(IRRCNN) [49], and a Capsule Network-based Framework (COVID-CAPS) [57].

Except for the pre-trained CNN models that are already presented in Table 4 and Table 5, several specialized diagnosing models using those pre-trained models researchers adopt nowadays to enlarge accuracy. I represent the researchers' proposed feature extraction method based on both CT images and X-ray images in Table 6. 
Table 6: Summary of specially designed architectures for COVID-19 detection

\begin{tabular}{|c|c|c|c|c|c|}
\hline $\begin{array}{l}\text { Feature } \\
\text { extraction } \\
\text { method }\end{array}$ & Papers & $\begin{array}{l}\text { Interpretability } \\
\text { method }\end{array}$ & $\begin{array}{l}\text { Dataset } \\
\text { Type }\end{array}$ & Classification & $\begin{array}{c}\text { Experimental } \\
\text { Result(\%) }\end{array}$ \\
\hline $\begin{array}{l}\text { Parallel-Dilated } \\
\text { COVIDNet } \\
\text { (PDCOVIDNet) }\end{array}$ & [1] & Grad-Cam & X-ray & Mult- class & Accuracy:96.58 \\
\hline CovMUNET & [3] & Not used & X-ray & Mult- class & Accuracy:99.41 \\
\hline COVID TV-UNet & {$[5]$} & Not used & CT & Mult- class & $\begin{array}{c}\text { Dice } \\
\text { Score: } 86 \%\end{array}$ \\
\hline COVID-SDNet & [13] & Grad-cam & X-ray & Binary & Accuracy:97.37 \\
\hline COVID-Net & {$[36]$} & Grad-cam & X-ray & Multi-class & Accuracy:93.30 \\
\hline CovXNet & [26] & Grad-cam & X-ray & Multi-class & Accuracy: 97.40 \\
\hline CheXNet & [35] & Not used & X-ray & Binary & Accuracy:95.90 \\
\hline CoroNet & [54] & Not used & X-ray & Binary & Accuracy:89.60 \\
\hline COVID-CAPS & {$[57]$} & Not used & X-ray & Binary & Accuracy:95.50 \\
\hline Decapse(Capsule) & {$[60]$} & Not used & CT & Binary & Accuracy: 87.60 \\
\hline
\end{tabular}

PDCOVIDNet detects COVID-19 from chest X-ray images. The proposed PDCOVIDNet differentiates the dilation rate in a parallel stack of convolution layers on $\mathrm{CNN}$, thus reflecting more distinguishable features. In paper [1] authors use a 2,905 chest X-ray image for diagnosing, from that 2,324 images are used for the training datasets.

CovMUNET is a multiple loss based deep neural network procedure that detects COVID-19 cases from X-ray images. In paper [3] using CovMUNET for 2 class classification (COVID vs non-COVID) researchers gained noticeable accuracy than 3- class classification (COVID-19 vs normal vs pneumonia) 99.41\% CovMUNET contains two branches namely Reconstruction Branch and 'Classification Branch' that calculate two different losses. In this study, the author utilizes 5 -fold cross-validation and the dataset size is 6594.

COVID TV-UNet is a deep learning-based framework for detecting pathologic COVID-19 regions or associated tissues in pulmonary CT images tissue areas from clinical CT images. In this study [5], the author uses a total of 929 images from publicly available COVID-19 segmentation datasets. among which 473 are labeled as COVID-19.

COVID Smart Data based Network (COVID-SDNet) is a CNN based classifier that incorporates segmentation, data-augmentation, and data transformations along with an eligible Convolutional Neural Network (CNN) for inference. The author introduces us with a high clinical quality dataset [13] named COVIDGR1.0 which comprises 754 images among which 377 is labeled as COVID-19. For a transfer learning approach, ResNet-50 is adopted by the researchers that are initialized with ImageNet weights. 
COVID-Net specifically proposes a neural network to detect COVID-19 using chest X-ray images. Based on the advantages of CXR imaging for the rapid outcome of COVID-19 screening, tangibility, mobility, the author makes predictions through the COVIDNet interpretability method. The author makes use of projection-expansion-projection design patterns in COVID-Net architecture [36]. The proposed COVID-Net was pre-trained on the ImageNet dataset and then assigned to the COVIDx dataset and achieved accuracy about $93.3 \%$ on the COVIDx dataset.

CovXNet is a multi-dilation convolutional neural network which detects COVID-19 and other pneumonia automatically using chest X-ray images that utilize depthwise convolution for efficiently extracting diversified features. In the paper[26] author uses a total of 6161 datasets among which the first dataset consists of 5856 images (1583 normal X-rays, 1493 non-COVID viral pneumonia X-rays, and 2780 bacterial pneumonia X-rays) and the 2nd dataset comprises 305 images.

ChexNet is basically a DenseNet-121 type of deep network trained on the ChestX-ray14 dataset. They used the Softmax activation function To classify COVID-19 the Softmax activation function is used(Normal, Viral Pneumonia, and Bacterial Pneumonia). The number of trainable parameters in this model is $6,955,906$. Before the classification layer, the author used pre-trained CheXNet to extract 1024-D feature vectors by taking the output after global pooling in paper [35]. The sensitivity achieved by ChexNet is noticeable. In this paper, the author utilizes the largest Covid-19 dataset named QaTa-Cov19 that constitutes 6286 images among them 5028 is used to train the dataset.

CoroNet is a Deep Convolutional Neural Network model that is based on Xception architecture. In paper [54] authors collect two different datasets that are publicly available then create their own dataset to detect COVID-19 infection automatically using chest X-ray images. In the paper the author also uses softmax to predict and it is inspected from that CoroNet has 33,969,964 parameters in total out of which $33,969,964$ trainable and 54528 are non-trainable parameters. Author achieves promising results through CoroNet in spite of using a small dataset(COVID-19 284, Normal 310).

COVID-CAPS is a Capsule Network-based Framework that identifies COVID-19 cases from X-ray Images which consists of 4 convolutional layers and 3 Capsule layers. It is noticeable COVID-CAPS shows higher performance dealing with a small dataset [57]. The number of trainable parameters using COVID-CAPS without pre-training and with pre-training is 295,488 .

Detail-Oriented Capsule Networks (DECAPS) combine Capsule Networks (CapsNets) that is basically based on ResNet to identify discriminative image features to detect COVID-19 patients using CT images. In paper [60] the author gained accuracy $87.60 \%$ using DECAPS model which comprises a smallest amount of dataset. Because of the scarcity of sample image authors applied conditional adversarial network and also adopted other preprocessing techniques for instance Rescaling(286X286), Cropping(256X256). Among the total dataset 391 images are labeled as COVID-19 and 339 images are labeled as Normal. ResNet has three residual blocks and, outcome 1024 feature maps, with a $1 \times 1$ convolutional layer. It contains a ReLU non-linear layer. Author also shows using Peekaboo with DECAPS accuracy of the diagnosing model increased.

\section{Classification}

The classification process took place in the softmax layer, and a fully connected layer whereas the Convolutional layer proceeds as a feature extractor in a pre-trained CNN. Some researchers proposed improvements based on pre-trained CNN along with a Support Vector Machine(SVM) classifier [46], [66]. The SVM classifier uses deep features that are extracted from each CNN network for detection. Researchers combine CNN with KNN and a support estimator network [14] that requires huge data to train. In paper [6] researchers use a COV-ELM classifier that classifies COVID-19 cases from the chest $x$-ray images using an extreme learning machine (ELM) and lessens training time with the least interventions required to tune the networks. 
Researchers developed an end to end web-based detection system with a Bagging trees classifier to replicate a digital clinical pipeline and ease the screening of suspicious cases [67]. Researchers achieved satisfactory performance despite the limited number of image samples using the Bagging trees classifier. In [34], researchers proposed Adaptive Feature Selection Guided Deep Forest(AFS-DF) as a classification method in the meanwhile they compared AFS-DF with Logistic Regression (LR), Random Forests (RF), Neural Networks (NN), SVM classifiers and AFS-DF attain higher accuracy.

It is obvious from Table 7 Binary class is mostly used by researchers than Multi-class. However, Binary classification may create ambiguity while detecting COVID-19 as it can not distinguish between other Viral Pneumonia and COVID-19.

Table 7: Classification Methods

\begin{tabular}{lll}
\hline $\begin{array}{c}\text { Classification } \\
\text { Methods }\end{array}$ & \multicolumn{1}{c}{ Papers } & Total \\
\hline \multirow{3}{*}{ Binary } & {$[4],[9],[11],[13],[14],[15],[18][19],[21],[22],[23],[25],[27],[28],[29],[30],[32],[34]$} & \\
& $,[35],[37],[42],[44],[46],[48],[$ 49],[ 51], [52],[ 53], [54],[ 55], & 40 \\
& {$[57],[58],[60],[62],[64],[66],[67],[68],[69],[72]$} & \\
Multi-class & {$[11],[2],[3],[5],[6],[7],[10],[12],[16],[$ 17], [22], [24],[ 31],[ 33], [36],[ 38], [ 40],[ } & \\
& $41],[42],[43],[45],[47],[49],[50],[56],[58],[61],[64],[70],[71]$, & 32 \\
& {$[73],[74]$} &
\end{tabular}

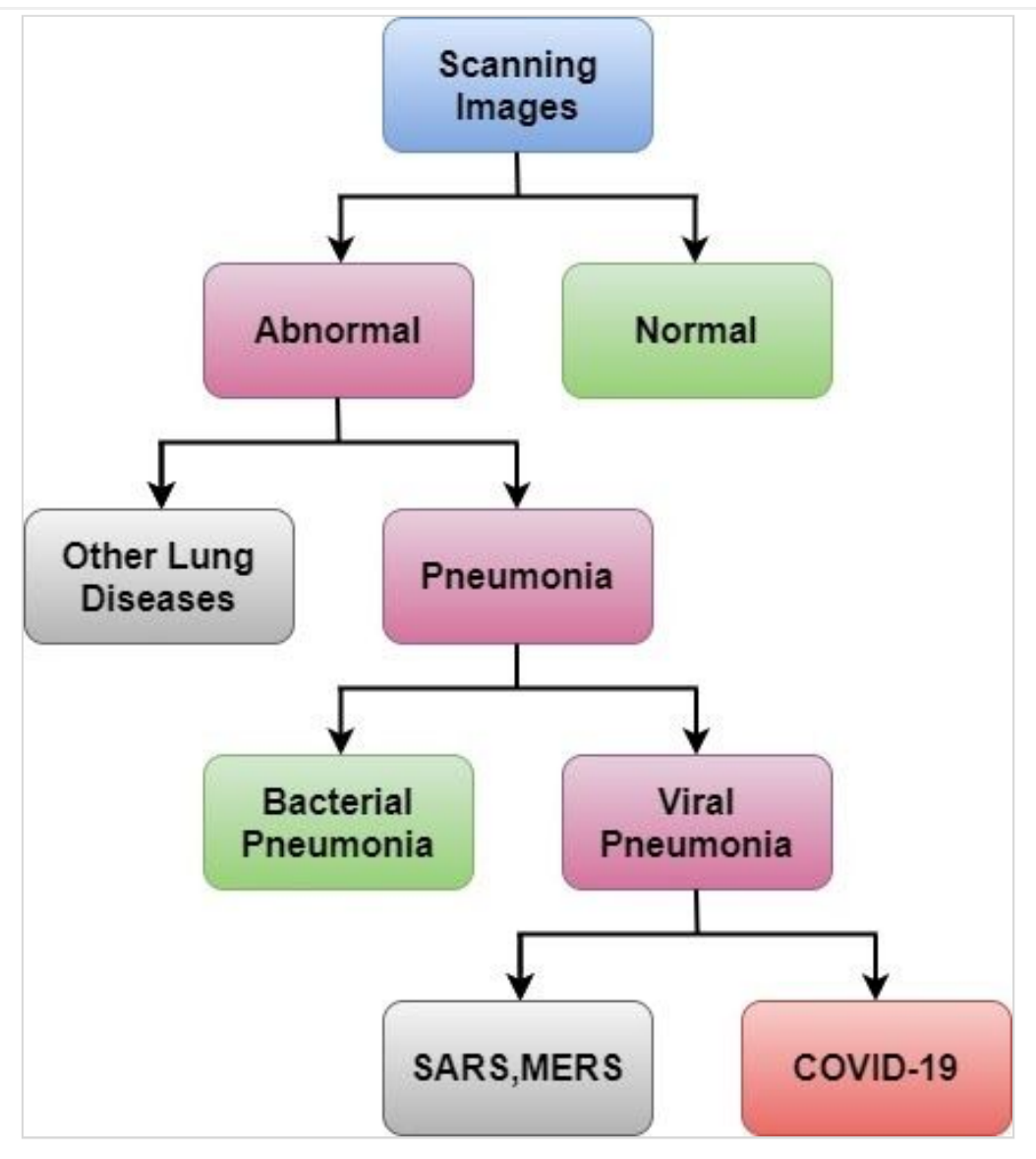

Figure 8: A relationship of Lung Diseases 
A straightforward way of COVID-19 diagnosing system tasks is binary classifying the scanning images into COVID-19 class and normal class, and that is adopted by many papers[21].[19],[23].As shown in Fig. 8., test images of other groups of abnormal Lung Diseases can be miscategorized as COVID-19.

Lung diseases belonging to the same subclass share similar patterns in diagnosing using X-ray image or CT images and contain a higher probability to be miscategorized to resolve this dilemma researchers adopt multi-class classification in many papers [7],[12],[17].

\section{Experimental results}

Several pre-trained models are proposed by the researchers; perhaps specific methods are enough qualified to gain the highest accuracy depending on its total dataset size, classifier, number of convolutional layers in a CNN, and so on. Experimental results are measured in terms of accuracy in this survey, when researchers don't state accuracy as an alternative sensitivity, Area Under Curve (AUC) or specificity is picked up that illustrates how precisely and accurately that model can predict the result.

\subsection{Experimental results for $\mathrm{CT}$ images}

Abbreviated evaluation of COVID-19 diagnosing models using CT images presented in Table 8. I also mentioned the size of the train, test, and validation set. Some authors in their papers [11],[53],[59] did not apparently provide the size of train, test, and validation set, I compute them with corresponding train, test, and validation split ratio. On the contrary, some papers apparently stated the size of the train-test-validation data of COVID-19 images [5],[68],[69] and for those papers, I computed the ratio according to the split that was provided. Even so, for some papers, the distribution of the dataset [50],[55],[34] was not clearly mentioned. Moreover, some papers apparently mentioned the use of data for validation[5],[8],[11] along with some papers do not state exact data instead provide a comparison based on 10-fold cross-validation[50], 5-fold cross-validation [53] for performance assessment. Along with the best experimental results using CT images feature extraction methods corresponding to the accuracy and total dataset(COVID-19 patients, non affected patients, and other pneumonia diseases) also reviewed in Table 8. In terms of sensitivity, accuracy, and AUC VGG, IRRCNN, ResNet scores higher 97\%, 99.56\% and $99.40 \%$ respectively.

Table 8: Summary of experimental result along with the extraction method using CT images

\begin{tabular}{|c|c|c|c|c|c|c|c|c|}
\hline $\begin{array}{l}\text { Paper } \\
\text { no }\end{array}$ & Date & $\begin{array}{c}\text { Total } \\
\text { images }\end{array}$ & $\begin{array}{c}\text { Train } \\
\text { Test Val } \\
\text { Ratio(\%) }\end{array}$ & Train & Test & Validation & Result(\%) & $\begin{array}{c}\text { Feature } \\
\text { Extraction for } \\
\text { best result }\end{array}$ \\
\hline 4 & $\begin{array}{l}27 \text { July } \\
2020\end{array}$ & 400 & $\begin{array}{l}\text { Train :80 } \\
\text { Test:20 }\end{array}$ & 329 & 80 & $\begin{array}{c}\text { Not } \\
\text { specified }\end{array}$ & $\begin{array}{c}\text { Accuracy: } \\
95.2 \%\end{array}$ & NasNetMobile \\
\hline 5 & $\begin{array}{l}27 \text { July } \\
2020\end{array}$ & 929 & $\begin{array}{c}\text { Train }: 70 \\
\text { Test:22 } \\
\text { Val:8 }\end{array}$ & 650 & 204 & 75 & $\begin{array}{c}\text { Dice } \\
\text { score: } 86\end{array}$ & TV-UNet \\
\hline 8 & 7 July 2020 & 829 & $\begin{array}{c}\text { Train:45 } \\
\text { Test:50 } \\
\text { Val:5 }\end{array}$ & 373 & 414 & 42 & $\begin{array}{c}\text { Dice } \\
\text { score:90 }\end{array}$ & $\begin{array}{l}\text { Region based } \\
\text { active learning }\end{array}$ \\
\hline 9 & $\begin{array}{l}10 \text { July } \\
2020\end{array}$ & 34006 & not clear & $\begin{array}{c}\text { Not } \\
\text { specified }\end{array}$ & $\begin{array}{c}\text { Not } \\
\text { specified }\end{array}$ & $\begin{array}{c}\text { Not } \\
\text { specified }\end{array}$ & $\begin{array}{l}\text { Sensitivity: } \\
96.7\end{array}$ & Inception \\
\hline 2 & $\begin{array}{l}29 \text { July } \\
2020\end{array}$ & 829 & $\begin{array}{c}\text { Train:36 } \\
\text { Test:8 }\end{array}$ & 300 & 73 & $\begin{array}{c}\text { Not } \\
\text { specified }\end{array}$ & $\begin{array}{c}\text { Specificity: } \\
99.97\end{array}$ & cGAN \\
\hline
\end{tabular}




\begin{tabular}{|c|c|c|c|c|c|c|c|c|}
\hline 11 & 7 July 2020 & 829 & $\begin{array}{c}\text { Test:45 } \\
\text { Test:50 } \\
\text { Val:5 }\end{array}$ & 373 & 414 & 42 & $\begin{array}{c}\text { Sensitivity: } \\
97\end{array}$ & VGG \\
\hline 12 & 2 June 2020 & 10250 & $\begin{array}{c}\text { Not } \\
\text { specified }\end{array}$ & $\begin{array}{c}\text { Not } \\
\text { specified }\end{array}$ & $\begin{array}{c}\text { Not } \\
\text { specified }\end{array}$ & $\begin{array}{c}\text { Not } \\
\text { specified }\end{array}$ & $\begin{array}{c}\text { Sensitivity: } \\
90.19\end{array}$ & ResNet \\
\hline 21 & $\begin{array}{c}17 \text { June } \\
2020\end{array}$ & 812 & $\begin{array}{l}\text { Train:42 } \\
\text { Test:40 } \\
\text { Val:18 }\end{array}$ & 341 & 325 & 146 & $\begin{array}{c}\text { Accuracy: } \\
79.50\end{array}$ & DenseNet \\
\hline 29 & $\begin{array}{l}24 \text { June } \\
2020\end{array}$ & 746 & $\begin{array}{l}\text { Train: } 52 \\
\text { Test: } 25 \\
\text { Val: } 14\end{array}$ & 425 & 203 & 118 & $\begin{array}{c}\text { Accuracy: } \\
90.61\end{array}$ & DenseNet \\
\hline 34 & 7 May 2020 & 2522 & $\begin{array}{c}\text { Not } \\
\text { specified }\end{array}$ & & & & $\begin{array}{c}\text { Accuracy: } \\
97.79\end{array}$ & AFS-DF \\
\hline 39 & $\begin{array}{c}21 \text { May } \\
2020\end{array}$ & 100 & $\begin{array}{l}\text { Train:45 } \\
\text { Test:50 } \\
\text { Val:5 }\end{array}$ & 45 & 50 & 5 & $\begin{array}{c}\text { Sensitivity: } \\
72.50\end{array}$ & ResNet \\
\hline 42 & $\begin{array}{c}28 \text { May } \\
2020\end{array}$ & 746 & $\begin{array}{l}\text { Train:55 } \\
\text { Test:22 } \\
\text { Val:23 }\end{array}$ & 410 & 164 & 172 & $\begin{array}{c}\text { Accuracy: } \\
99.40\end{array}$ & ResNet \\
\hline 45 & 6 April 2020 & $\begin{array}{c}\text { Dataset } \\
\text { 1:1865 }\end{array}$ & $\begin{array}{l}\text { Random } \\
\text { split }\end{array}$ & 1725 & 270320 & $\begin{array}{c}\text { Not } \\
\text { specified }\end{array}$ & $\begin{array}{l}\text { AUC: } \\
99.40\end{array}$ & ResNet \\
\hline 46 & 7 April 2020 & $\begin{array}{l}6000 \\
6000\end{array}$ & $\begin{array}{l}\text { Train:75 } \\
\text { Test:25 }\end{array}$ & 4500 & 1500 & & $\begin{array}{c}\text { Accuracy: } \\
98.27\end{array}$ & $\begin{array}{l}\text { Fusing and } \\
\text { ranking deep } \\
\text { features }\end{array}$ \\
\hline 49 & $\begin{array}{c}10 \text { April } \\
2020\end{array}$ & 420 & $\begin{array}{l}\text { Train:45 } \\
\text { Test:10 } \\
\text { Val:45 }\end{array}$ & 189 & 42 & 189 & $\begin{array}{l}\text { Accuracy: } \\
99.56\end{array}$ & $\begin{array}{c}\text { Inception } \\
\text { Recurrent } \\
\text { Residual } \\
\text { Neural Network } \\
\text { (IRRCNN) }\end{array}$ \\
\hline 50 & $\begin{array}{c}12 \text { April } \\
2020\end{array}$ & 110. & $\begin{array}{l}\text { Val:10 } \\
\text { fold } \\
\text { cross }\end{array}$ & & & & $\begin{array}{c}\text { Accuracy: } \\
89.00\end{array}$ & U-Net \\
\hline 53 & $\begin{array}{c}14 \text { April } \\
2020\end{array}$ & 360 & $\begin{array}{l}\text { Train:80 } \\
\text { Test:10 } \\
\text { Val:10 }\end{array}$ & 288 & 36 & 36 & $\begin{array}{c}\text { Accuracy: } \\
89.20\end{array}$ & VGG \\
\hline 55 & $\begin{array}{l}15 \text { April } \\
2020\end{array}$ & 3,855 & $\begin{array}{c}\text { Not } \\
\text { specified }\end{array}$ & & & & $\begin{array}{c}\text { Sensitivity: } \\
95.00\end{array}$ & ResNet \\
\hline 58 & $\begin{array}{l}17 \text { April } \\
2020\end{array}$ & 746 & $\begin{array}{l}\text { Train:57 } \\
\text { Test:28 } \\
\text { Val:15 }\end{array}$ & 425 & 208 & 119 & $\begin{array}{c}\text { Accuracy: } \\
83.00\end{array}$ & DenseNet \\
\hline 59 & $\begin{array}{c}17 \text { April } \\
2020\end{array}$ & 470 & $\begin{array}{l}\text { Train:60 } \\
\text { Test:40 }\end{array}$ & 282 & 188 & $\begin{array}{c}\text { Not } \\
\text { specified }\end{array}$ & $\begin{array}{c}\text { Accuracy: } \\
93.65\end{array}$ & $\begin{array}{l}\text { FFT-Gabor } \\
\text { scheme }\end{array}$ \\
\hline 60 & $\begin{array}{l}17 \text { April } \\
2020\end{array}$ & 746 & $\begin{array}{l}\text { Train:85 } \\
\text { Test:15 }\end{array}$ & 634 & 112 & $\begin{array}{c}\text { Not } \\
\text { specified }\end{array}$ & $\begin{array}{c}\text { Accuracy: } \\
87.60\end{array}$ & Decapse \\
\hline 63 & $\begin{array}{c}21 \text { April } \\
2020\end{array}$ & 829 & $\begin{array}{l}\text { Train:80 } \\
\text { Test:20 }\end{array}$ & 663 & 166 & $\begin{array}{c}\text { Not } \\
\text { specified }\end{array}$ & $\begin{array}{c}\text { Sensitivity: } \\
86.70\end{array}$ & U-Net \\
\hline
\end{tabular}




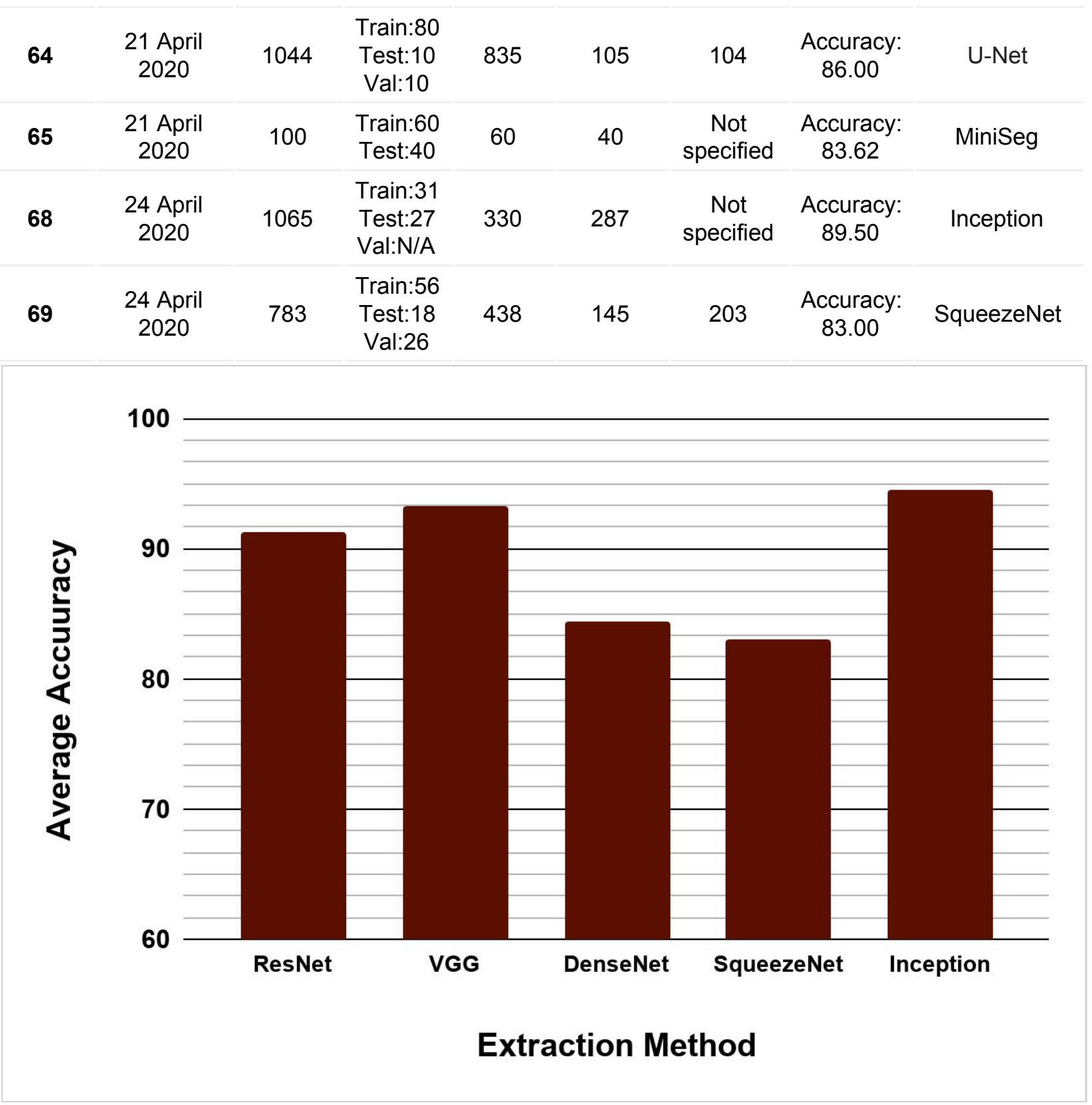

Figure 9: Average accuracy

In this section, the average result is reviewed over the extraction method. It is sharply clear from Fig. 9 that the average accuracy of Inception is far exceeding above $90 \%$ than the other 4 models and the average accuracy of SqueezeNet is not up to the mark.

\subsection{Experimental results for $\mathrm{X}$-ray images}

Abbreviated evaluation of COVID-19 diagnosing models using X-ray images presented in Table 9.I also stated the size of the train, test, and validation set. Some authors in their papers [19],[18] did not apparently provide the size of the train, test, and validation set, I compute them with corresponding train, test, and validation split ratio. On the contrary, some papers apparently stated the size of the train-test-validation data of COVID-19 images [16],[20],[22] and for those papers, I computed the ratio 
according to the split that was provided. Even so, for some papers, the distribution of the dataset [7],[10],[26] was not clearly mentioned. Moreover, some papers apparently mentioned the use of data for validation[1],[16],[25],[30] along with some papers do not state exact data instead provide a comparison based on 10 -fold cross-validation[6], 5 -fold cross-validation $[3,[18]$ for performance assessment.

Along with the best experimental results using X-ray images feature extraction methods corresponding to the accuracy or other metrics and total dataset(COVID-19 patients, non affected patients, and other pneumonia diseases) also reviewed in Table 9.In terms of accuracy and AUC [14],[72] performance of DenseNet is up to the mark $99.49 \%, 88.04 \%$ respectively whereas NasNetLarge scores $100 \%$ sensitivity [23], f1 score of FFT is $95 \%$. Additionally in respect of accuracy Xception+ResNet [61] is significantly the highest scorer $99.56 \%$.

Table 9: Summary of experimental result along with method using X-ray images

\begin{tabular}{|c|c|c|c|c|c|c|c|c|}
\hline $\begin{array}{l}\text { Paper } \\
\text { no }\end{array}$ & Date & $\begin{array}{c}\text { Total } \\
\text { images }\end{array}$ & $\begin{array}{c}\text { Train Test } \\
\text { Val Ratio(\%) }\end{array}$ & Train & Test & Validation & Result(\%) & $\begin{array}{c}\text { Feature } \\
\text { Extraction for } \\
\text { best result }\end{array}$ \\
\hline 1 & $\begin{array}{l}30 \text { July } \\
2020\end{array}$ & 2,905 & $\begin{array}{l}\text { Train:80 } \\
\text { Test:10 } \\
\text { Val:10 }\end{array}$ & 2324 & 291 & 291 & $\begin{array}{l}\text { Accuracy } \\
: 96.58\end{array}$ & PDCOVIDNet \\
\hline 3 & $\begin{array}{l}29 \text { July } \\
2020\end{array}$ & 6,594 & 5 fold Val & & & & $\begin{array}{c}\text { Accuracy } \\
: 99.41\end{array}$ & ComMUNET \\
\hline 4 & $\begin{array}{l}27 \text { July } \\
2020\end{array}$ & 400 & $\begin{array}{l}\text { Train:80 } \\
\text { Test:20 }\end{array}$ & 320 & 80 & & $\begin{array}{l}\text { Accuracy: } \\
95.4-100\end{array}$ & NasNetMobile \\
\hline 6 & $\begin{array}{l}16 \mathrm{Jul} \\
2020\end{array}$ & 9,521 & $\begin{array}{c}10-\text { fold } \\
\text { cross-Val }\end{array}$ & & & & $\begin{array}{l}\text { f1 score: } \\
95.00\end{array}$ & FFT \\
\hline 7 & $\begin{array}{l}16 \text { July } \\
2020\end{array}$ & 2,186 & $\begin{array}{c}\text { 5-fold } \\
\text { cross-validati } \\
\text { on }\end{array}$ & & & & $\begin{array}{l}\text { Accuracy: } \\
98.00\end{array}$ & ResNet \\
\hline 10 & $\begin{array}{l}13 \text { July } \\
2020\end{array}$ & 375 & $\begin{array}{l}\text { Train:80 } \\
\text { Test:10 } \\
\text { Val:10 }\end{array}$ & 300 & 38 & 37 & $\begin{array}{c}\text { Accuracy: } \\
97.3\end{array}$ & VGG16 \\
\hline 13 & $\begin{array}{l}2 \text { June } \\
2020\end{array}$ & 754 & $\begin{array}{l}\text { Train:80 } \\
\text { Test:20 }\end{array}$ & 603 & 150 & & $\begin{array}{l}\text { Accuracy: } \\
97.37\end{array}$ & ResNet \\
\hline 14 & $\begin{array}{l}7 \text { June } \\
2020\end{array}$ & 5824 & $\begin{array}{l}\text { Train:80 } \\
\text { Test:20 }\end{array}$ & 4659 & 1165 & & $\begin{array}{c}\text { Accuracy } \\
: 99.49\end{array}$ & DenseNet \\
\hline 15 & $\begin{array}{c}8 \text { June } \\
2020\end{array}$ & 1419 & $\begin{array}{l}\text { Train:80 } \\
\text { Test:20 }\end{array}$ & 1135 & 284 & & $\begin{array}{l}\text { Accuracy: } \\
98.94\end{array}$ & Xception \\
\hline 16 & $\begin{array}{l}9 \text { June } \\
2020\end{array}$ & 6523 & $\begin{array}{l}\text { Train:30 } \\
\text { Test:30 } \\
\text { Val:40 }\end{array}$ & 2000 & 2000 & 2523 & $\begin{array}{l}\text { Accuracy: } \\
98.00\end{array}$ & VGG \\
\hline 17 & $\begin{array}{l}9 \text { June } \\
2020\end{array}$ & 1262 & $\begin{array}{l}\text { Train: } 88 \\
\text { Test:12 }\end{array}$ & 1100 & 162 & & $\begin{array}{l}\text { Sensitivity: } \\
90.74\end{array}$ & AlexNet \\
\hline 18 & $\begin{array}{c}10 \text { June } \\
2020\end{array}$ & 1302 & $\begin{array}{l}\text { Train } 60 \\
\text { Test40 }\end{array}$ & 781 & 521 & & $\begin{array}{l}\text { Precision: } \\
88.90 \\
\text { Sensitivity: } \\
85.10\end{array}$ & DenseNet \\
\hline
\end{tabular}




\begin{tabular}{|c|c|c|c|c|c|c|c|c|}
\hline 19 & $\begin{array}{l}11 \text { June } \\
2020\end{array}$ & 8474 & $\begin{array}{l}\text { Train:90 } \\
\text { Test:10 }\end{array}$ & 7626 & 847 & & $\begin{array}{c}\text { Accuracy: } \\
98.60\end{array}$ & VGG \\
\hline 20 & $\begin{array}{l}16 \text { June } \\
2020\end{array}$ & 15282 & $\begin{array}{l}\text { Train:90 } \\
\text { Test:10 }\end{array}$ & 13,703 & 1579 & & $\begin{array}{c}\text { Accuracy: } \\
98.06\end{array}$ & ResNet \\
\hline 22 & $\begin{array}{c}18 \text { june } \\
2020\end{array}$ & 30099 & $\begin{array}{l}\text { Train:88 } \\
\text { Test:11 } \\
\text { Val:N/A }\end{array}$ & 26487 & 3310 & & $\begin{array}{l}\text { Accuracy: } \\
98.00\end{array}$ & $\begin{array}{c}\text { NASNet } \\
\text { Large }\end{array}$ \\
\hline 23 & $\begin{array}{c}18 \text { June } \\
2020\end{array}$ & 3309 & $\begin{array}{l}\text { Train:80 } \\
\text { Test:20 }\end{array}$ & 2647 & 662 & & $\begin{array}{c}\text { Sensitivity: } \\
100.00\end{array}$ & NASNet Large \\
\hline 24 & $\begin{array}{l}18 \text { June } \\
2020\end{array}$ & 35500 & $\begin{array}{c}\text { Train:88 } \\
\text { Test:4 } \\
\text { Val:8 }\end{array}$ & 31340 & 1800 & 2360 & $\begin{array}{c}\text { Accuracy: } \\
98.00\end{array}$ & Inception \\
\hline 25 & $\begin{array}{l}19 \text { June } \\
2020\end{array}$ & 1312 & $\begin{array}{l}\text { Train:70 } \\
\text { Test:20 } \\
\text { Val:10 }\end{array}$ & 918 & 263 & 131 & $\begin{array}{c}\text { Accuracy: } \\
97.40\end{array}$ & CAD system \\
\hline 26 & $\begin{array}{l}20 \text { June } \\
2020\end{array}$ & 6161 & $\begin{array}{c}\text { 5-fold } \\
\text { cross-validati } \\
\text { on }\end{array}$ & & & & $\begin{array}{l}\text { Accuracy: } \\
97.40\end{array}$ & CovXNet \\
\hline 27 & $\begin{array}{l}23 \text { June } \\
2020\end{array}$ & 50,000 & $\begin{array}{l}\text { Train:70 } \\
\text { Test:30 }\end{array}$ & 35000 & 15,000 & & $\begin{array}{c}\text { Accuracy: } \\
97.54\end{array}$ & ResNet \\
\hline 28 & $\begin{array}{l}23 \text { June } \\
2020\end{array}$ & 2271 & $\begin{array}{l}\text { Train:70 } \\
\text { Test:30 }\end{array}$ & 1590 & 681 & & $\begin{array}{c}\text { Accuracy: } \\
98.9\end{array}$ & DenseNet \\
\hline 30 & $\begin{array}{l}26 \text { June } \\
2020\end{array}$ & 239 & $\begin{array}{l}\text { Train: } 70 \\
\text { Test: } 20 \\
\text { Val: } 10\end{array}$ & 167 & 20 & 22 & $\begin{array}{c}\text { Accuracy: } \\
98\end{array}$ & $\begin{array}{c}\text { Residual Att } \\
\text { Net }\end{array}$ \\
\hline 31 & $\begin{array}{c}1 \text { May } \\
2020\end{array}$ & 6297 & $\begin{array}{c}\text { Train :27 } \\
\text { Test :26 } \\
\text { Val :46 }\end{array}$ & 1591 & 1439 & 2772 & $\begin{array}{c}\text { Accuracy: } \\
97.10\end{array}$ & DenseNet \\
\hline 32 & $\begin{array}{c}5 \text { May } \\
2020\end{array}$ & 502 & $\begin{array}{l}\text { Train:70 } \\
\text { Test: } 20 \\
\text { Val: } 10\end{array}$ & 399 & 100 & 3 & $\begin{array}{l}\text { Accuracy: } \\
88.90\end{array}$ & ResNet \\
\hline 33 & $\begin{array}{c}6 \text { May } \\
2020\end{array}$ & 1144 & $\begin{array}{l}\text { Train:70 } \\
\text { Test:30 }\end{array}$ & 801 & 343 & & $\begin{array}{l}\text { F1 Score: } \\
89.60\end{array}$ & Inception \\
\hline 35 & $\begin{array}{c}8 \text { May } \\
2020\end{array}$ & 6286 & $\begin{array}{l}\text { Train:80 } \\
\text { Test:20 }\end{array}$ & 5029 & 1257 & & $\begin{array}{l}\text { Accuracy: } \\
95.90\end{array}$ & CheXNet \\
\hline 36 & $\begin{array}{c}11 \text { May } \\
2020\end{array}$ & 13,975 & $\begin{array}{c}\text { Not } \\
\text { specified }\end{array}$ & & & & $\begin{array}{c}\text { Accuracy: } \\
93.30\end{array}$ & COVID-NET \\
\hline 37 & $\begin{array}{l}16 \text { May } \\
2020\end{array}$ & 50 & $\begin{array}{c}\text { Not } \\
\text { specified }\end{array}$ & & & & $\begin{array}{c}\text { Accuracy: } \\
\text { 90-92 }\end{array}$ & VGG \\
\hline 38 & $\begin{array}{l}17 \text { May } \\
2020\end{array}$ & 1764 & $\begin{array}{l}\text { Train:70 } \\
\text { Test:30 }\end{array}$ & 1235 & 530 & & $\begin{array}{c}\text { Accuracy: } \\
95.12\end{array}$ & ResNet \\
\hline 40 & $\begin{array}{l}21 \text { May } \\
2020\end{array}$ & 2239 & $\begin{array}{c}\text { Not } \\
\text { specified }\end{array}$ & & & & $\begin{array}{c}\text { Accuracy } \\
: 97.01\end{array}$ & DCSL \\
\hline
\end{tabular}




\begin{tabular}{|c|c|c|c|c|c|c|c|c|}
\hline 41 & $\begin{array}{c}23 \text { May } \\
2020\end{array}$ & 701 & $\begin{array}{l}\text { Train:80 } \\
\text { Test:20 }\end{array}$ & 561 & 140 & & $\begin{array}{c}\text { Accuracy: } \\
98.22\end{array}$ & Inception \\
\hline 43 & $\begin{array}{l}1 \text { April } \\
2020\end{array}$ & 3905 & $\begin{array}{l}10 \text {-fold cross } \\
\text { validation }\end{array}$ & & & & $\begin{array}{c}\text { Accuracy: } \\
99.18\end{array}$ & MobileNet \\
\hline 44 & $\begin{array}{l}2 \text { April } \\
2020\end{array}$ & 5824 & $\begin{array}{l}\text { Train:80 } \\
\text { Test:20 }\end{array}$ & 4659 & 1165 & & $\begin{array}{c}\text { Accuracy: } \\
99.00\end{array}$ & Resnet \\
\hline 47 & $\begin{array}{l}9 \text { April } \\
2020\end{array}$ & 16995 & $\begin{array}{l}5 \text {-fold cross } \\
\text { validation }\end{array}$ & & & & $\begin{array}{c}\text { Accuracy: } \\
94.80\end{array}$ & DenseNet \\
\hline 48 & $\begin{array}{l}9 \text { April } \\
2020\end{array}$ & 414 & $\begin{array}{l}\text { Train:70 } \\
\text { Test:15 } \\
\text { Val: } 15\end{array}$ & 290 & 62 & 62 & $\begin{array}{l}\text { Accuracy: } \\
98.00\end{array}$ & DenseNet \\
\hline 49 & $\begin{array}{l}10 \text { April } \\
2020\end{array}$ & 5216 & $\begin{array}{l}\text { Train:80 } \\
\text { Test:10 } \\
\text { Val:10 }\end{array}$ & 4172 & 522 & 522 & $\begin{array}{c}\text { Accuracy: } \\
94.52\end{array}$ & $\begin{array}{c}\text { (IRRCNN) } \\
\text { and NABLA-3 }\end{array}$ \\
\hline 51 & $\begin{array}{l}13 \text { April } \\
2020\end{array}$ & 455 & $\begin{array}{l}10 \text {-fold cross } \\
\text { validation }\end{array}$ & & & & $\begin{array}{c}\text { Accuracy: } \\
91.24\end{array}$ & $\begin{array}{r}\text { (Resnet-50+V } \\
\text { GG16+CNN) }\end{array}$ \\
\hline 52 & $\begin{array}{l}13 \text { April } \\
2020\end{array}$ & 537 & $\begin{array}{l}\text { Train:70 } \\
\text { Test:20 } \\
\text { Val:10 }\end{array}$ & 376 & 107 & 54 & $\begin{array}{c}\text { Accuracy: } \\
93.5\end{array}$ & MobileNetv2 \\
\hline 54 & $\begin{array}{l}14 \text { April } \\
2020\end{array}$ & 1300 & $\begin{array}{c}\text { 4-fold } \\
\text { validation }\end{array}$ & & & & $\begin{array}{c}\text { Accuracy: } \\
89.60\end{array}$ & Xception \\
\hline 56 & $\begin{array}{l}16 \text { April } \\
2020\end{array}$ & 16700 & $\begin{array}{l}\text { Train:90 } \\
\text { Test:10 }\end{array}$ & 15030 & 1670 & & $\begin{array}{c}\text { Accuracy: } \\
99.01\end{array}$ & Inception \\
\hline 57 & $\begin{array}{l}16 \text { April } \\
2020\end{array}$ & 864 & $\begin{array}{l}\text { Train:90 } \\
\text { Val:10 }\end{array}$ & 777 & & 87 & $\begin{array}{c}\text { Accuracy: } \\
95.70\end{array}$ & COVID-CAPS \\
\hline 61 & $\begin{array}{l}17 \text { April } \\
2020\end{array}$ & 15085 & $\begin{array}{l}\text { Train:25\% } \\
\text { val: } 75 \%\end{array}$ & 3783 & & 11302 & $\begin{array}{c}\text { Accuracy: } \\
99.56\end{array}$ & $\begin{array}{c}\text { Xception+Res } \\
\text { Net }\end{array}$ \\
\hline 62 & $\begin{array}{l}20 \text { April } \\
2020\end{array}$ & $\begin{array}{c}5071 \\
(5536 \\
\text { after } \\
\text { augment } \\
\text { ation) }\end{array}$ & $\begin{array}{l}\text { Train:40 } \\
\text { Test:60 }\end{array}$ & 2028 & 30426 & & $\begin{array}{l}\text { Sensitivity } \\
: 97.50\end{array}$ & ResNet \\
\hline 66 & $\begin{array}{l}22 \text { April } \\
2020\end{array}$ & 381 & $\begin{array}{l}\text { Train:60 } \\
\text { Test:20 } \\
\text { Val:20 }\end{array}$ & 228 & 76 & 76 & $\begin{array}{c}\text { Accuracy: } \\
95.33\end{array}$ & ResNet \\
\hline 67 & $\begin{array}{l}22 \text { April } \\
2020\end{array}$ & 274 & $\begin{array}{c}10 \text {-fold } \\
\text { cross } \\
\text { validation }\end{array}$ & & & & $\begin{array}{c}\text { Accuracy: } \\
99.00\end{array}$ & DenseNet \\
\hline 70 & $\begin{array}{c}24 \text { April } \\
2020\end{array}$ & 109203 & $\begin{array}{l}5 \text {-fold cross } \\
\text { validation }\end{array}$ & & & & $\begin{array}{c}\text { Accuracy: } \\
95.30\end{array}$ & $\begin{array}{c}\text { Domain } \\
\text { Extension } \\
\text { Transfer } \\
\text { Learning CNN }\end{array}$ \\
\hline 71 & $\begin{array}{l}28 \text { April } \\
2020\end{array}$ & 13800 & $\begin{array}{l}\text { Train:98 } \\
\text { Test:2 }\end{array}$ & 13,525 & 276 & & $\begin{array}{c}\text { Accuracy: } \\
93.90\end{array}$ & EfficientNet \\
\hline
\end{tabular}




\begin{tabular}{ccccccccc}
\hline 72 & $\begin{array}{c}30 \text { April } \\
2020\end{array}$ & 59937 & $\begin{array}{c}\text { Train:80 } \\
\text { Test:20 }\end{array}$ & 47,950 & 11987 & & $\begin{array}{c}\text { AUC: } \\
88.04\end{array}$ & DenseNet \\
\hline 73 & $\begin{array}{c}30 \mathrm{Apr} \\
2020\end{array}$ & 11663 & $\begin{array}{c}\text { Train:91 } \\
\text { Test:9 }\end{array}$ & 10613 & 1050 & & $\begin{array}{c}\text { Sensitivity: } \\
88.33\end{array}$ & ResNet \\
\hline 74 & $\begin{array}{c}30 \mathrm{Apr} \\
2020\end{array}$ & 15111 & $\begin{array}{c}\text { Train :80 } \\
\text { Test:10 } \\
\text { Val: } 10\end{array}$ & 12088 & 1511 & 1511 & $\begin{array}{c}\text { Accuracy: } \\
89.40\end{array}$ & DenseNet \\
\hline
\end{tabular}

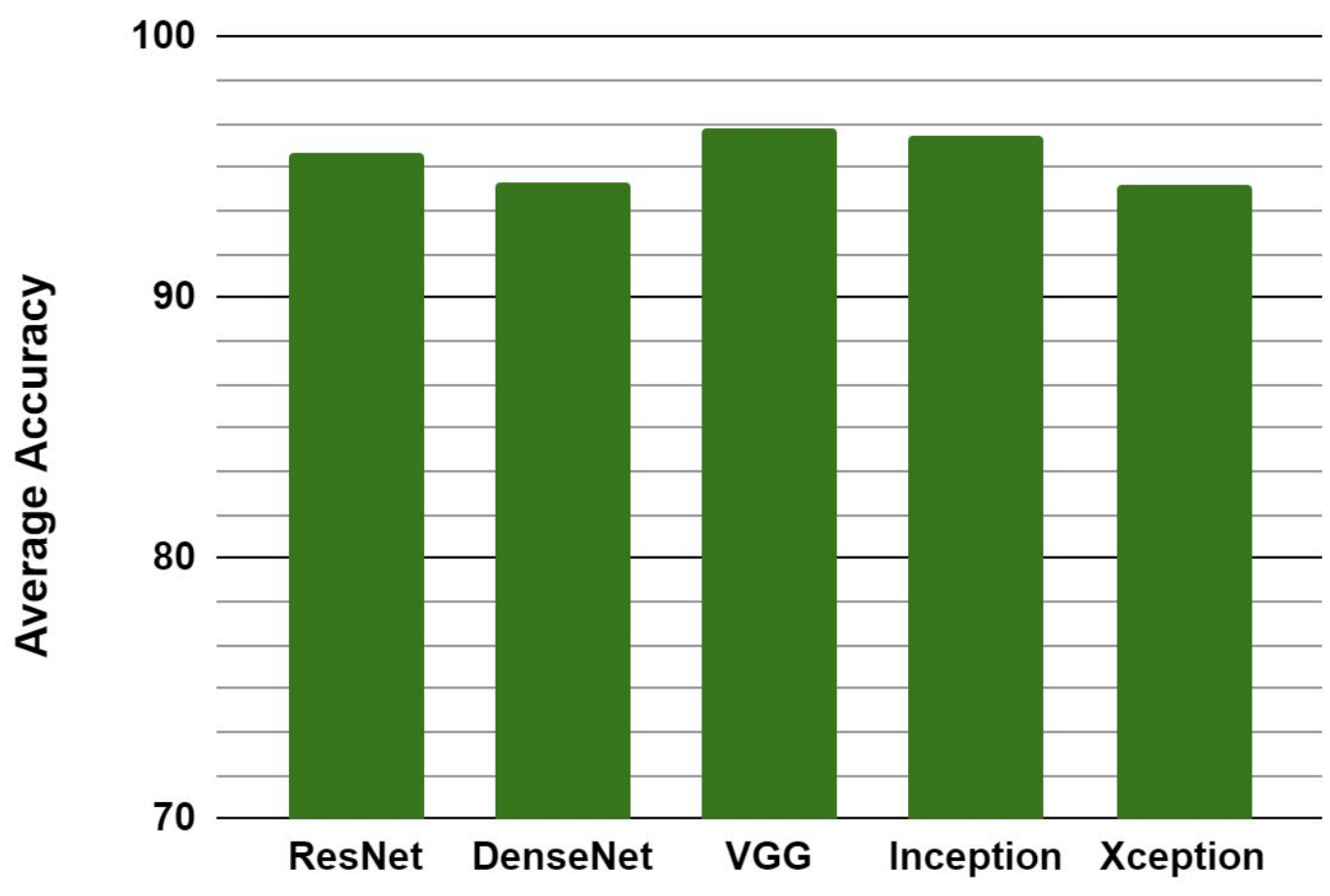

Extraction Method

Figure 10: Average Accuracy for X-ray image

In this section, average accuracy is reviewed over the best 5 extraction methods. It is sharply clear from Fig. 10 that the average accuracy of VGG is far exceeding above $95 \%$ than the other 4 models and the average accuracy of Xception is not up to the mark. The average accuracy of VGG and Inception is immensely close $96.5 \%, 96.3 \%$ individually. X-ray image based diagnosing is superior rather than CT image-based diagnosing clearly appreciable from Fig. 9 and Fig.10

\section{Interpretability}

Class activation mapping is a method to generate heatmaps of images that is a visualization and can be interpreted as telling researchers wherein the image the neural net is (metaphorically) looking to make its decision indicating a highly important area. Several variations of the method including Score-CAM and Grad-CAM (Gradient Weighted Class Activation Mapping) is accepted widely.CAM is proposed by researchers to examine overfitting occurrence even CAM is capable to classify relevant portions of the image for CNN. Actually, when the diagnosing model has significant accuracy on the training data, but 
unmarked accuracy on the Test dataset, CAM helps to verify whether the CNN is biased or not while predicting on the features of the images.

Perhaps, because of its nonlinearity vanishing property of the classifiers nowadays Gradient Weighted Class Activation Mapping (Grad-CAM) [47] is broadly by researchers. As an interpretability method gradient-guided class activation maps (Grad-CAM++) and layerwise relevance propagation (LRP), Local Interpretable Model-Agnostic Explanations (LIME) are widely adopted by many researchers for explaining the predictions and to identify the critical regions on patient's chest besides generating class-discriminating attention maps.

However, In this survey, I oversee two interpretability methods that are Grad-Cam and CAM. Both the X-ray image based diagnosing model and CT-image based diagnosing model used Grad-Cam more in comparison to CAM. Besides in some literature [4],[22] authors also utilize LIME with the purpose of rectifying misclassification. Chest X-ray images of a patient In Fig. 11 are displayed to output heatmaps for interpretation of the ultimate result and represent an intuitive understanding of which area is the model focusing on.

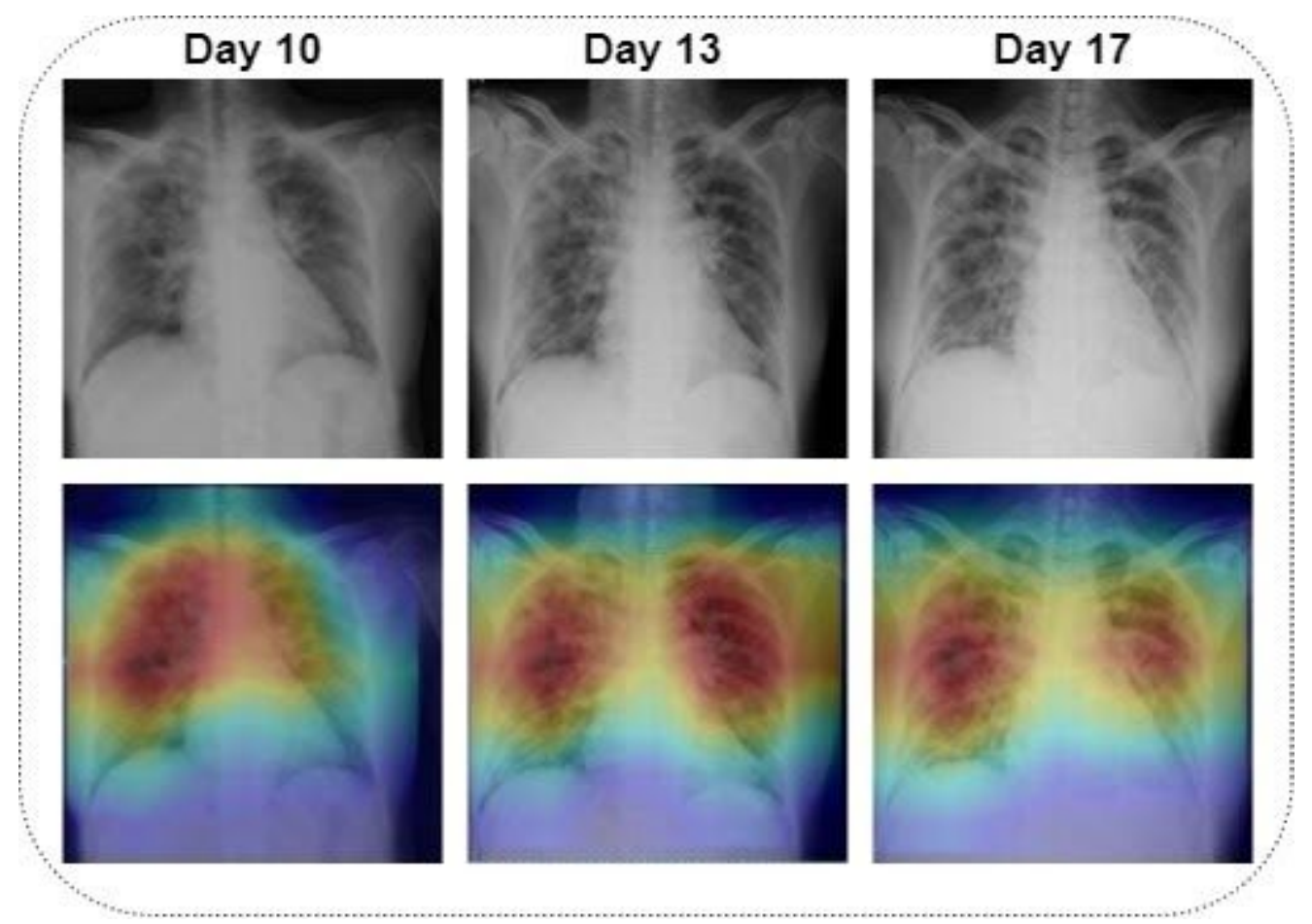

Figure 11: Chest X-ray images of a patent in three points in the upper row and in a lower row their heatmaps using Grad-Cam

In CT image-based diagnosing model researchers use Grad-Cam at 3 literature, CAM at 2 literature depicted in Table 10.

Table 10: Interpretability methods used in the CT images based works

\begin{tabular}{ccc}
\hline Interpretability method & Papers & Total \\
\hline Grad-Cam & {$[12],[45],[58]$} & 3 \\
\hline Cam & {$[53],[69]$} & 2 \\
\hline
\end{tabular}


In the X-ray image-based diagnosing model researchers use Grad-Cam at 15 literature, CAM at 4 literature depicted in Table 11.

Table 11: Interpretability methods used in the X-ray images based works

\begin{tabular}{ccc}
\hline Interpretability method & Papers & Total \\
\hline & {$[1],[6],[10],[13],[15],[16],[20],[26],[31],[32],[47]$,} & \\
Grad-Cam & {$[52],[56],[70],[73]$} & 15 \\
\hline Cam & {$[22],[41],[72],[74]$} & 4 \\
\hline
\end{tabular}

The entire use of Grad-Cam and Cam based on both CT-image and X-ray image based diagnosing systems in this survey are interpreted in Fig. 12 to deliver a sharp concept of interpretability.

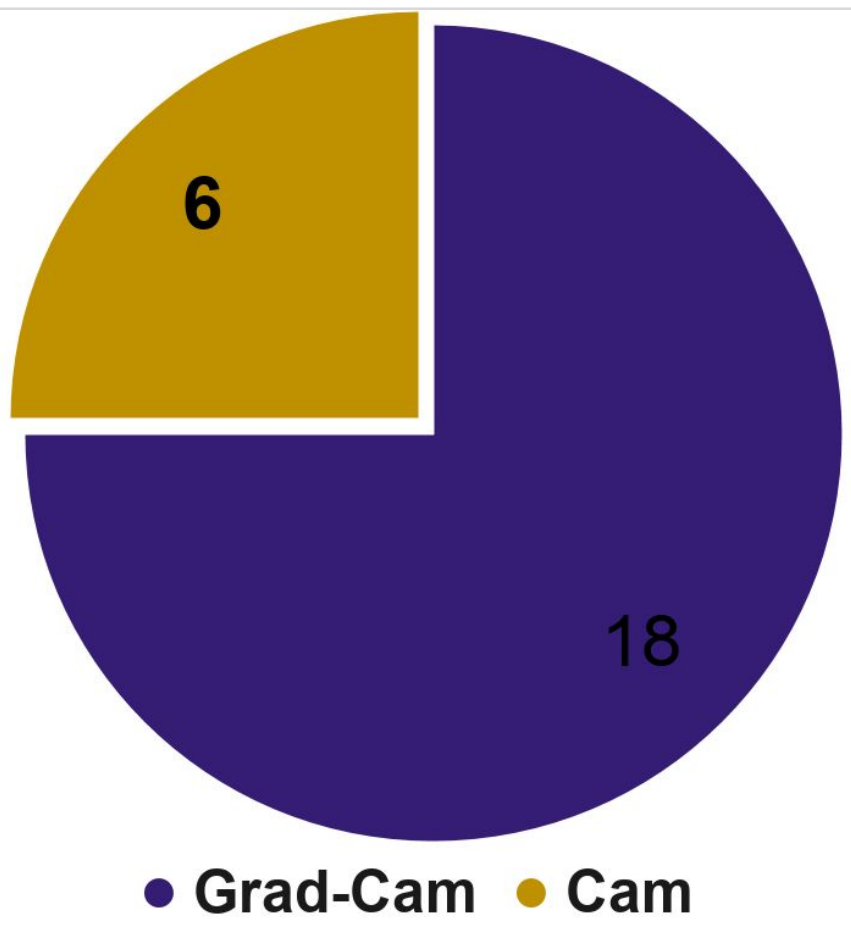

Figure 12: Total number of Grad-Cam and CAM for both CT and X-ray based works

\section{Discussion}

In this survey, I reviewed 74 automatic COVID-19 diagnosing models and overseen the characteristics of this diagnosing model. It is obvious from this survey, the average accuracy of X-ray image based 
diagnosing is better than CT image-based diagnosing $96.5 \%, 94.5 \%$ respectively. The reason could be a massive amount of X-ray training data than CT training data. The average training data size of the CT image-based diagnosing system is 609.9 whereas the average training data size of the X-ray image-based diagnosing model is 2857.9. But many authors don't clarify their dataset size [10], as well as many authors, do not provide a clear indication of training dataset size in their literature [36],[40].

For transfer learning, some diagnosing models adopted pre-trained CNN model [46] even showed high performance $98.27 \%$ accuracy, while others proposed various architectures to detect COVID-19 cases from Chest X-ray images to obtain more discriminative features as in paper [3] author propose CovMUNET, which is a multiple loss deep neural network approach, in paper [25] author proposed a deep learning CAD system to simultaneously recognize the COVID-19 9 among the other eight lung diseases: Atelectasis, Infiltration, Pneumothorax, Mass, Effusion, Pneumonia, Cardiomegaly, and Nodule using chest X-ray images, in paper [53] author propose a weakly supervised deep learning strategy for detecting and classifying COVID-19 infection from CT images. The proposed model can reduce the demands of manual labeling of CT images. But still, researchers are unable to detect infection and discriminate COVID-19 from non-COVID-19 cases. To enhance the performance of the classifiers, researchers proposed[16] multi-class classification. We also abbreviated the results of diagnosing models. Some of the models report very good performance, but the size of some test sets are not sufficient [19].

\section{Conclusion}

In this survey, I provide the overview of research works that detect COVID-19 using CT image and X-ray image on the basis of feature extraction method, classification, interpretability, and model accuracy. Early quarantine is a must for an affected person in order to decrease the infection rate and to get clinical support diagnosing COVID-19 as early as possible is mandatory. Al-based diagnosing models using Chest X-ray images and CT-Scan images have a higher potentiality to support radiologists in COVID-19 detection rapidly.

The rate of death can be decreased by recognizing patients that are affected so that they can get immediate medical attention and that is possible only by adopting Al-based diagnosing systems as it can diagnose at a very limited time and precisely. It is noticeable in this survey that we analyze different research work so that researchers can pick out the best one or discover any model based on our data that we analyze considering different aspects. I hope this survey can provide valuable insight into computer vision efforts against the COVID-19 pandemic and assist the researchers in new work.

\section{References}

1. Chowdhury NK, Rahman M, Kabir MA. PDCOVIDNet: A Parallel-Dilated Convolutional Neural Network Architecture for Detecting COVID-19 from Chest X-ray Images. arXiv preprint arXiv:2007.14777. 2020 Jul 29.

2. Jiang Y, Chen H, Loew M, Ko H. COVID-19 CT Image Synthesis with a Conditional Generative Adversarial Network. arXiv preprint arXiv:2007.14638. 2020 Jul 29.

3. Sayyed AQ, Saha D, Hossain AR. CovMUNET: A Multiple Loss Approach towards Detection of COVID-19 from Chest X-ray. arXiv preprint arXiv:2007.14318. 2020 Jul 28.

4. Ahsan MM, Gupta KD, Islam MM, Sen S, Rahman M, Hossain MS. Study of Different Deep Learning Approach with Explainable Al for Screening Patients with COVID-19 Symptoms: Using CT Scan and Chest X-ray Image Dataset. arXiv preprint arXiv:2007.12525. 2020 Jul 24 
5. Saeedizadeh N, Minaee S, Kafieh R, Yazdani S, Sonka M. COVID TV-UNet: Segmenting COVID-19 Chest CT Images Using Connectivity Imposed U-Net. arXiv preprint arXiv:2007.12303. 2020 Jul 24.

6. Rajpal S, Kumar N, Rajpal A. COV-ELM classifier: An Extreme Learning Machine based identification of COVID-19 using Chest-ray Images. arXiv preprint arXiv:2007.08637. 2020 Jul 16.

7. Al-Timemy AH, Khushaba RN, Mosa ZM, Escudero J. An Efficient Mixture of Deep and Machine Learning Models for COVID-19 and Tuberculosis Detection Using X-ray Images in Resource Limited Settings. arXiv preprint arXiv:2007.08223. 2020 Jul 16.

8. Laradji I, Rodriguez P, Branchaud-Charron F, Lensink K, Atighehchian P, Parker W, Vazquez D, Nowrouzezahrai D. A Weakly Supervised Region-Based Active Learning Method for COVID-19 Segmentation in CT Images. arXiv preprint arXiv:2007.07012. 2020 Jul 7.

9. Kumar R, Khan AA, Zhang S, Wang W, Abuidris Y, Amin W, Kumar J. Blockchain-Federated-Learning and Deep Learning Models for COVID-19 detection using CT Imaging. arXiv preprint arXiv:2007.06537. 2020 Jul 10.

10. Soares LP, Soares CP. Automatic Detection of COVID-19 Cases on X-ray images Using Convolutional Neural Networks. arXiv preprint arXiv:2007.05494. 2020 Jul 2.

11. Laradji I, Rodriguez P, Manas O, Lensink K, Law M, Kurzman L, Parker W, Vazquez D, Nowrouzezahrai D. A Weakly Supervised Consistency-based Learning Method for COVID-19 Segmentation in CT Images. arXiv preprint arXiv:2007.02180. 2020 Jul 4.

12. Jin C, Chen W, Cao Y, Xu Z, Zhang X, Deng L, Zheng C, Zhou J, Shi H, Feng J. Development and Evaluation of an AI System for COVID-19 Diagnosis. medRxiv. 2020 Jan 1.

13. Tabik S, Gómez-Ríos A, Martín-Rodríguez JL, Sevillano-García I, Rey-Area M, Charte D, Guirado E, Suárez JL, Luengo J, Valero-González MA, García-Villanova P. COVIDGR dataset and COVID-SDNet methodology for predicting COVID-19 based on Chest X-ray images. arXiv preprint arXiv:2006.01409. 2020 Jun 2.

14. Ahishali M, Degerli A, Yamac M, Kiranyaz S, Chowdhury ME, Hameed K, Hamid T, Mazhar R, Gabbouj M. A Comparative Study on Early Detection of COVID-19 from Chest X-ray Images. arXiv preprint arXiv:2006.05332. 2020 Jun 7.

15. Singh KK, Siddhartha M, Singh A. Diagnosis of Coronavirus Disease (COVID-19) from Chest X-ray images using modified XceptionNet. ROMANIAN JOURNAL OF INFORMATION SCIENCE AND TECHNOLOGY. 2020 Jan 1;23:S91-105.

16. Brunese L, Mercaldo F, Reginelli A, Santone A. Explainable deep learning for pulmonary disease and coronavirus COVID-19 detection from X-rays. Computer Methods and Programs in Biomedicine. 2020 Jun 20:105608.

17. Salih SQ, Abdulla HK, Ahmed ZS, Surameery NM, Rashid RD. Modified AlexNet Convolution Neural Network For Covid-19 Detection Using Chest X-ray Images. Kurdistan Journal of Applied Research. 2020 Jun 9:119-30.

18. Chatterjee S, Saad F, Sarasaen C, Ghosh S, Khatun R, Radeva P, Rose G, Stober S, Speck O, Nürnberger A. Exploration of Interpretability Techniques for Deep COVID-19 Classification using Chest X-ray Images. arXiv preprint arXiv:2006.02570. 2020 Jun 3. 
19. Heidari M, Mirniaharikandehei S, Khuzani AZ, Danala G, Qiu Y, Zheng B. Improving performance of CNN to predict likelihood of COVID-19 using chest X-ray images with preprocessing algorithms. arXiv preprint arXiv:2006.12229. 2020 Jun 11.

20. Liu B, Yan B, Zhou Y, Yang Y, Zhang Y. Experiments of Federated Learning for COVID-19 Chest X-ray Images. arXiv preprint arXiv:2007.05592. 2020 Jul 5.

21. Yang X, He X, Zhao J, Zhang Y, Zhang S, Xie P. COVID-CT-Dataset: A CT Image Dataset about COVID-19. arXiv preprint arXiv:2003.13865. 2020 Mar.

22. Punn NS, Agarwal S. Automated diagnosis of COVID-19 with limited posteroanterior chest X-ray images using fine-tuned deep neural networks. arXiv preprint arXiv:2004.11676. 2020 Apr 23.

23. Boudrioua MS. COVID-19 detection from chest X-ray images using CNNs models: Further evidence from Deep Transfer Learning. Available at SSRN 3630150. 2020 Jun 18.

24. Asif S, Wenhui $Y$, Jin H, Tao Y, Jinhai S. Classification of COVID-19 from Chest X-ray images using Deep Convolutional Neural Networks. medRxiv. 2020 Jan 1.

25. Al-antari MA, Hua CH, Lee S. Fast Deep Learning Computer-Aided Diagnosis against the Novel COVID-19 pandemic from Digital Chest X-ray Images.

26. Mahmud T, Rahman MA, Fattah SA. CovXNet: A multi-dilation convolutional neural network for automatic COVID-19 and other pneumonia detection from chest X-ray images with transferable multi-receptive feature optimization. Computers in biology and medicine. 2020 Jun 20:103869.

27. Abbas A, Abdelsamea MM, Gaber M. 4S-DT: Self Supervised Super Sample Decomposition for Transfer learning with application to COVID-19 detection. arXiv preprint arXiv:2007.11450. 2020 Jun 26.

28. Shelke A, Inamdar M, Shah V, Tiwari A, Hussain A, Chafekar T, Mehendale N. Chest X-ray classification using Deep learning for automated COVID-19 screening. medRxiv. 2020 Jan 1.

29. Saeedi A, Saeedi M, Maghsoudi A. A novel and reliable deep learning web-based tool to detect COVID-19 infection form chest CT-scan. arXiv preprint arXiv:2006.14419. 2020 Jun 24.

30. Sharma V, Dyreson C. COVID-19 detection using Residual Attention Network an Artificial Intelligence approach. arXiv preprint arXiv:2006.16106. 2020 Jun 26.

31. Lv D, Qi W, Li Y, Sun L, Wang Y. A cascade network for Detecting COVID-19 using chest X-rays. arXiv preprint arXiv:2005.01468. 2020 May 1.

32. Oh Y, Park S, Ye JC. Deep learning covid-19 features on cxr using limited Train data sets. IEEE Transactions on Medical Imaging. 2020 May 8.

33. Pereira RM, Bertolini D, Teixeira LO, Silla Jr CN, Costa YM. COVID-19 identification in chest $\mathrm{X}$-ray images on flat and hierarchical classification scenarios. Computer Methods and Programs in Biomedicine. 2020 May 8:105532.

34. Sun L, Mo Z, Yan F, Xia L, Shan F, Ding Z, Shao W, Shi F, Yuan H, Jiang H, Wu D. Adaptive Feature Selection Guided Deep Forest for COVID-19 Classification with Chest CT. arXiv preprint arXiv:2005.03264. 2020 May 7. 
35. Yamac M, Ahishali M, Degerli A, Kiranyaz S, Chowdhury ME, Gabbouj M. Convolutional Sparse Support Estimator Based Covid-19 Recognition from X-ray Images. arXiv preprint arXiv:2005.04014. 2020 May 8.

36. Wang L, Wong A. COVID-Net: A Tailored Deep Convolutional Neural Network Design for Detection of COVID-19 Cases from Chest X-ray Images. arXiv preprint arXiv:2003.09871. 2020 Mar 22.

37. Manapure P, Likhar K, Kosare H. Detecting COVID-19 in X-ray images with Keras, Tensor Flow, and Deep Learning. Assessment.;2:3.

38. Abbas A, Abdelsamea MM, Gaber MM. Classification of COVID-19 in chest X-ray images using DeTraC deep convolutional neural network. arXiv preprint arXiv:2003.13815. 2020 Mar 26.

39. Fan DP, Zhou T, Ji GP, Zhou Y, Chen G, Fu H, Shen J, Shao L. Inf-Net: Automatic COVID-19 Lung Infection Segmentation from CT Images. IEEE Transactions on Medical Imaging. 2020 May 22.

40. Li T, Han Z, Wei B, Zheng Y, Hong Y, Cong J. Robust Screening of COVID-19 from Chest X-ray via Discriminative Cost-Sensitive Learning. arXiv preprint arXiv:2004.12592. 2020 Apr 27.

41. Tahir A, Qiblawey Y, Khandakar A, Rahman T, Khurshid U, Musharavati F, Kiranyaz S, Chowdhury ME. Coronavirus: Comparing COVID-19, SARS and MERS in the eyes of Al. arXiv preprint arXiv:2005.11524. 2020 May 23.

42. Ahuja S, Panigrahi BK, Dey N, Rajinikanth V, Gandhi TK. Deep transfer learning-based automated detection of COVID-19 from lung CT scan slices.

43. Apostolopoulos ID, Aznaouridis SI, Tzani MA. Extracting possibly representative COVID-19 Biomarkers from X-ray images with Deep Learning approach and image data related to Pulmonary Diseases. Journal of Medical and Biological Engineering. 2020 May 14:1.

44. Khalifa NE, Taha MH, Hassanien AE, Elghamrawy S. Detection of coronavirus (COVID-19) associated pneumonia based on generative adversarial networks and a fine-tuned deep transfer learning model using chest X-ray dataset. arXiv preprint arXiv:2004.01184. 2020 Apr 2.

45. Gozes O, Frid-Adar M, Sagie N, Zhang H, Ji W, Greenspan H. Coronavirus detection and analysis on chest ct with deep learning. arXiv preprint arXiv:2004.02640. 2020 Apr 6.

46. Ozkaya U, Ozturk S, Barstugan M. Coronavirus (COVID-19) Classification using Deep Features Fusion and Ranking Technique. arXiv preprint arXiv:2004.03698. 2020 Apr 7.

47. Karim MR, Döhmen T, Rebholz-Schuhmann D, Decker S, Cochez M, Beyan O. DeepCOVIDExplainer: Explainable COVID-19 Diagnosis Based on Chest X-ray Images.

48. aEzzat D, Ella HA. GSA-DenseNet121-COVID-19: a hybrid deep learning architecture for the diagnosis of COVID-19 disease based on gravitational search optimization algorithm. arXiv preprint arXiv:2004.05084. 2020 Apr 9.

49. Alom MZ, Rahman MM, Nasrin MS, Taha TM, Asari VK. COVID_MTNet: COVID-19 Detection with Multi-Task Deep Learning Approaches. arXiv preprint arXiv:2004.03747. 2020 Apr 7.

50. Chen X, Yao L, Zhang Y. Residual Attention U-Net for Automated Multi-Class Segmentation of COVID-19 Chest CT Images. arXiv preprint arXiv:2004.05645. 2020 Apr 12. 
51. Hall LO, Paul R, Goldgof DB, Goldgof GM. Finding covid-19 from chest x-rays using deep learning on a small dataset. arXiv preprint arXiv:2004.02060. 2020 Apr 5.

52. Li X, Li C, Zhu D. COVID-MobileXpert: On-Device COVID-19 Screening using Snapshots of Chest X-ray. arXiv preprint arXiv:2004.03042. 2020.

53. Hu S, Gao Y, Niu Z, Jiang Y, Li L, Xiao X, Wang M, Fang EF, Menpes-Smith W, Xia J, Ye H. Weakly supervised deep learning for covid-19 infection detection and classification from ct images. IEEE Access. 2020 Jun 29.

54. Khan Al, Shah JL, Bhat MM. Coronet: A deep neural network for detection and diagnosis of COVID-19 from chest x-ray images. Computer Methods and Programs in Biomedicine. 2020 Jun $5: 105581$.

55. Wu YH, Gao SH, Mei J, Xu J, Fan DP, Zhao CW, Cheng MM. JCS: An explainable COVID-19 diagnosis system by joint classification and segmentation. arXiv preprint arXiv:2004.07054. 2020 Apr 15.

56. Rajaraman S, Siegelman J, Alderson PO, Folio LS, Folio LR, Antani SK. Iteratively Pruned Deep Learning Ensembles for COVID-19 Detection in Chest X-rays. arXiv preprint arXiv:2004.08379. 2020 Apr 16.

57. Afshar P, Heidarian S, Naderkhani F, Oikonomou A, Plataniotis KN, Mohammadi A. Covid-caps: A capsule network-based framework for identification of covid-19 cases from x-ray images. arXiv preprint arXiv:2004.02696. 2020 Apr 6.

58. He X, Yang X, Zhang S, Zhao J, Zhang Y, Xing E, Xie P. Sample-Efficient Deep Learning for COVID-19 Diagnosis Based on CT Scans. medRxiv. 2020 Jan 1.

59. Al-Karawi D, Al-Zaidi S, Polus N, Jassim S. Machine Learning Analysis of Chest CT Scan Images as a Complementary Digital Test of Coronavirus (COVID-19) Patients. medRxiv. 2020 Jan 1.

60. Mobiny A, Cicalese PA, Zare S, Yuan P, Abavisani M, Wu CC, Ahuja J, de Groot PM, Van Nguyen H. Radiologist-Level COVID-19 Detection Using CT Scans with Detail-Oriented Capsule Networks. arXiv preprint arXiv:2004.07407. 2020 Apr 16.

61. Rahimzadeh M, Attar A. A New Modified Deep Convolutional Neural Network for Detecting COVID-19 from X-ray Images. arXiv preprint arXiv:2004.08052. 2020 Apr 17.

62. Minaee S, Kafieh R, Sonka M, Yazdani S, Soufi GJ. Deep-covid: Predicting covid-19 from chest X-ray images using deep transfer learning. arXiv preprint arXiv:2004.09363. 2020 Apr 20.

63. Zhou T, Canu S, Ruan S. An automatic COVID-19 CT segmentation network using spatial and channel attention mechanism. arXiv preprint arXiv:2004.06673. 2020 Apr.

64. Amyar A, Modzelewski R, Ruan S. Multi-task Deep Learning Based CT Imaging Analysis For COVID-19: Classification and Segmentation. medRxiv. 2020 Jan 1.

65. Qiu Y, Liu Y, Xu J. MiniSeg: An Extremely Minimum Network for Efficient COVID-19 Segmentation. arXiv preprint arXiv:2004.09750. 2020 Apr 21.

66. Sethy PK, Behera SK. Detection of coronavirus disease (covid-19) based on deep features. Preprints. 2020 Mar 19;2020030300:2020. 
67. Kassani SH, Kassasni PH, Wesolowski MJ, Schneider KA, Deters R. Automatic Detection of Coronavirus Disease (COVID-19) in X-ray and CT Images: A Machine Learning-Based Approach. arXiv preprint arXiv:2004.10641. 2020 Apr 22.

68. Wang S, Kang B, Ma J, Zeng X, Xiao M, Guo J, Cai M, Yang J, Li Y, Meng X, Xu B. A deep learning algorithm using CT images to screen for Corona Virus Disease (COVID-19). MedRxiv. 2020 Jan 1.

69. Polsinelli M, Cinque L, Placidi G. A Light CNN for detecting COVID-19 from CT scans of the chest. arXiv preprint arXiv:2004.12837. 2020 Apr 24.

70. Basu S, Mitra S. Deep Learning for Screening COVID-19 using Chest X-ray Images. arXiv preprint arXiv:2004.10507. 2020 Apr 22.

71. Luz EJ, Silva PL, Silva R, Silva L, Moreira G, Menotti D. Towards an Effective and Efficient Deep Learning Model for COVID-19 Patterns Detection in X-ray Images. CoRR. 2020.

72. Yeh CF, Cheng HT, Wei A, Liu KC, Ko MC, Kuo PC, Chen RJ, Lee PC, Chuang JH, Chen CM, Chou NK. A Cascaded Learning Strategy for Robust COVID-19 Pneumonia Chest X-ray Screening. arXiv preprint arXiv:2004.12786. 2020 Apr 24.

73. Zhang Y, Niu S, Qiu Z, Wei Y, Zhao P, Yao J, Huang J, Wu Q, Tan M. COVID-DA: Deep Domain Adaptation from Typical Pneumonia to COVID-19. arXiv preprint arXiv:2005.01577. 2020 Apr 30.

74. Goodwin BD, Jaskolski C, Zhong C, Asmani H. Intra-model Variability in COVID-19 Classification Using Chest X-ray Images. arXiv preprint arXiv:2005.02167. 2020 Apr 30. 